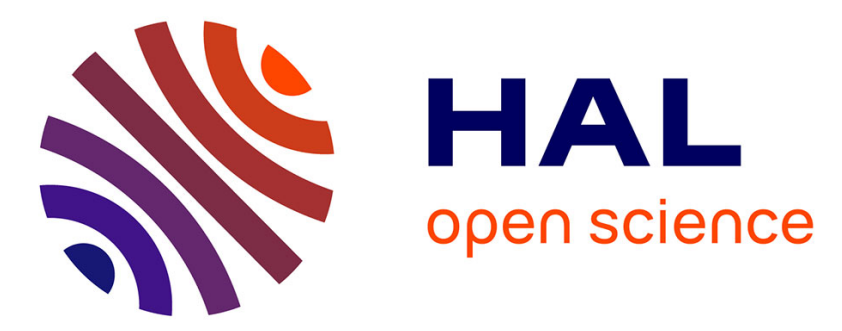

\title{
Wireless Ventilation Control for Large-Scale Systems: the Mining Industrial Case
}

Emmanuel Witrant, Alessandro d'Innocenzo, Guillaume Sandou, Fortunato

Santucci, Maria Domenica Di Benedetto, Alf J. Isaksson, Karl Henrik

Johansson, Silviu-Iulian Niculescu, Sorin Olaru, Emmanuele Serra, et al.

\section{To cite this version:}

Emmanuel Witrant, Alessandro d'Innocenzo, Guillaume Sandou, Fortunato Santucci, Maria Domenica Di Benedetto, et al.. Wireless Ventilation Control for Large-Scale Systems: the Mining Industrial Case. International Journal of Robust and Nonlinear Control, 2010, 20 (2), pp.226-251. 10.1002/rnc.1485 . hal-00448406

\section{HAL Id: hal-00448406 https://hal.science/hal-00448406}

Submitted on 18 Jan 2010

HAL is a multi-disciplinary open access archive for the deposit and dissemination of scientific research documents, whether they are published or not. The documents may come from teaching and research institutions in France or abroad, or from public or private research centers.
L'archive ouverte pluridisciplinaire HAL, est destinée au dépôt et à la diffusion de documents scientifiques de niveau recherche, publiés ou non, émanant des établissements d'enseignement et de recherche français ou étrangers, des laboratoires publics ou privés. 


\title{
Wireless Ventilation Control for Large-Scale Systems: the Mining Industrial Case
}

\author{
E. Witrant ${ }^{1, *}$, A. D'Innocenzo ${ }^{2}$, G. Sandou ${ }^{3}$, F. Santucci ${ }^{2}$, \\ M. D. Di Benedetto ${ }^{2}$, A. J. Isaksson ${ }^{4}$, K. H. Johansson ${ }^{5}$, S.-I. Niculescu ${ }^{6}$, \\ S. Olaru ${ }^{3}$, E. Serra ${ }^{2}$, S. Tennina ${ }^{2}$ and U. Tiberi ${ }^{2}$. \\ 1 Control Systems Department, Université Joseph Fourier / GIPSA-lab, Grenoble, France. \\ 2 Department of Electrical and Computer Engineering, DEWS - University of L'Aquila, L'Aquila, Italy. \\ 3 Automatic Control Department, SUPELEC, Gif sur Yvette, France. \\ 4 ABB Corporate Research, Vasterås, Sweden. \\ 5 Automatic Control Department, KTH, Stockholm, Sweden. \\ 6 LSS-SUPELEC, Gif-sur-Yvette, France.
}

\begin{abstract}
SUMMARY
This paper describes a new industrial case on automation, for large scale systems with high environmental impact: the mining ventilation control systems. Ventilation control is essential for the operation of a mine in terms of safety ( $C O$ and $N O_{x}$ regulation) and energy optimization. We first discuss a novel regulation architecture, highlighting the interest for a model-based control approach and the use of distributed sensing capabilities thanks to a wireless sensor network (WSN). We propose a new model for underground ventilation. The main components of the system dynamics are described with time-delays, transmission errors, energy losses and concentration profiles. Two different modelbased control approaches, which can embody the complex dynamics of the system, are proposed. The first one resorts to a nonlinear model predictive control strategy (receding horizon) and aims to energy minimization thanks to a continuous operation of the fans. The second one, based on a hybrid description of the model and fans operation, provides automatic verification of the wireless control thanks to abstraction techniques. These control strategies are compared with simulations, in terms of regulation efficiency, energy consumption and need for computational capabilities. The industrial case description and control strategies open new vistas for the development of global system approaches that allow for the optimization of energy consumption of complex large-scale systems. Copyright (c) 2008 John Wiley \& Sons, Ltd.
\end{abstract}

KEY WORDS: Network controlled systems, ventilation control, large scale systems, distributed flow control, wireless sensor networks.

*Correspondence to: E. Witrant, GIPSA-lab, Control Systems Department, ENSE ${ }^{3}$ - Domaine Universitaire BP46, 38402 Saint Martin d'Hères - Cedex, FRANCE, emmanuel.witrant@gipsa-lab.grenoble-inp.fr. 


\section{INTRODUCTION}

Mining ventilation is an interesting example of a large scale system with high environmental impact where advanced control strategies can bring major improvements. Indeed, one of the first objectives of modern mining industry is to fulfill environmental specifications [1] during the ore extraction and crushing, by optimizing the energy consumption or the production of polluting agents. The mine electric consumption was $4 \%$ of total industrial electric demand in the US in 1994 (6\% in 2007 in South Africa) and $90 \%$ of it was related to motor system energy [2]. Another interesting figure is given in [3] where it is estimated that the savings associated with global control strategies for fluid systems (pumps, fans and compressors) represent approximately $20 \%$ of the total manufacturing motor system energy savings. This motivates the development of new control strategies for large scale aerodynamic processes based on appropriate automation and a global consideration of the system. More specifically, the challenge in this paper is focused on the mining ventilation since as much as $50 \%$ or more of the energy consumed by the mining process may go into the ventilation (including heating the air). It is clear that investigating automatic control solutions and minimizing the amount of pumped air to save energy consumption (proportional to the cube of airflow quantity [4]) is of great environmental and industrial interest.

In the advanced ventilation control context depicted so far, reconfigurability and wireless interconnection are key automation components. The first issue is related to the variable topology of the mine (mobile process industry): after all accessible ore has been retrieved from a mine level, the extraction rooms are filled and a new level further down along the decline (which is a spiraling tunnel used by the trucks to reach the surface) is bored. All equipments, including the ventilation, have to be moved and re-configured in the new level. While making easier re-configurability, the second issue comes also from the blasting and drilling operations in the extraction rooms, rendering the wiring infeasible in these areas. Our approach strongly relies on distributed sensing and actuation capabilities, and consequently communication capabilities. Wireless networks can also be used for improving the efficiency of other processes that are of importance in operating a mine, e.g., equipment (trucks and ventilation system) maintenance, people and equipment localization, voice communication and security. We will consequently quickly discuss the wireless automation in the mining environment and determine the impact of such network on the controlled dynamics. Note that the specific study of wireless communications in underground areas is a specific field of research [5] out of the scope of this paper, where we focus on the ventilation control aspects. Nevertheless, further details on wireless network architecture and performance can be found in $[6]$ and [7].

We focus our attention on a specific control problem: the regulation of the underground fans. A new model is proposed to describe this part of the system, highlighting the main dynamics that should be considered for model-based control strategies. These dynamics involve communication constraints (delays and packet losses) associated with the WSN, energy losses and physical delays due to flow transport, and the dynamics of the stratified flow in the extraction room. While experimental results are not available yet to validate the proposed model, we tried to capture the fundamental dynamics that affect the closed-loop performances and represent them in a detailed simulation scenario.

Based on the previous model, two different control strategies are proposed to regulate the gas concentrations in the extraction rooms, essentially distinguished by continuous and 
hybrid automation. The first one is a nonlinear model-predictive control (receding horizon) scheme, which is particularly well suited to handle the model nonlinearities and to optimize a performance criterion (energy minimization) under some constraints. The second strategy is based on a conservative mathematical model of the system, and proposes a simple threshold control strategy. The advantage of this approach is that the control can be easily implemented and is suited for embedded fan controllers and pervasive sensors deployment in the rooms.

This work integrates the main results presented in $[8,7,9,10]$ in a unified way, highlighting the use of a WSN in the regulation process and the non-linear (continuous and hybrid) control aspects. The main novelty is the comparison between the continuous (nonlinear model predictive control) and the discrete approaches.

The paper is organized as follows. In Section 2, we illustrate and discuss in detail the mining ventilation control problem from an industrial point of view. In Section 3, we propose a mathematical model of the system. Section 4 is focused on a nonlinear model predictive control algorithm, while in Section 5 we introduce a conservative mathematical model of the system and formally prove safety of a threshold control strategy. These two control approaches are compared in Section 6.

\section{MINING VENTILATION CONTROL}

Traditionally, the control of large-scale systems is performed locally with decentralized pre-set control actions. The terms control and optimization consequently refer to the preliminary design of the global system and automation devices, without feedback or real-time control considerations. Mining ventilation provides an interesting example for the automation evolution of large-scale systems. To this end we have explored the literature background and found that we can track mathematical modeling efforts back to 1968 [11], when a steady-state compartmental model was proposed for flow networks with complex topology. In 1973, new results on the experimental determination of turbulent diffusion coefficients were presented [12], indicating a motivation for more advanced models. We have to wait until the 90's to find the first use of Navier-Stokes equations, with simplified chamber-like [13] and general mine aerology [14] models. The associated problems of nonlinearity and non stationary behavior, high dimensionality and numerical issues are quickly reported in [15]. This short literature overview also illustrates the parallel evolution of computing capability and automation complexity.

Mining engineers have been primarily concerned with proper design of ventilation tunnels, fans and turbines, and more generally with the short and long term planning of ventilation requirements [16]. However, recent technological developments in the fields of embedded control and wireless sensing [17, 18] systems motivate some new research efforts, seeking global automation strategies that would allow for an optimized real-time control of the ventilation system. The novel socio-political concern for energy consumption and increase of electricity prices in a context of worldwide competition also motivate theoretical research leading to improved control architectures, such as the one presented in this section.

\subsection{The mining system and main control objectives}

The global mine configuration is illustrated in Fig. 1. The ventilation is first achieved by a turbine and a heater connected on the surface to a vertical shaft. The heater is required (in 


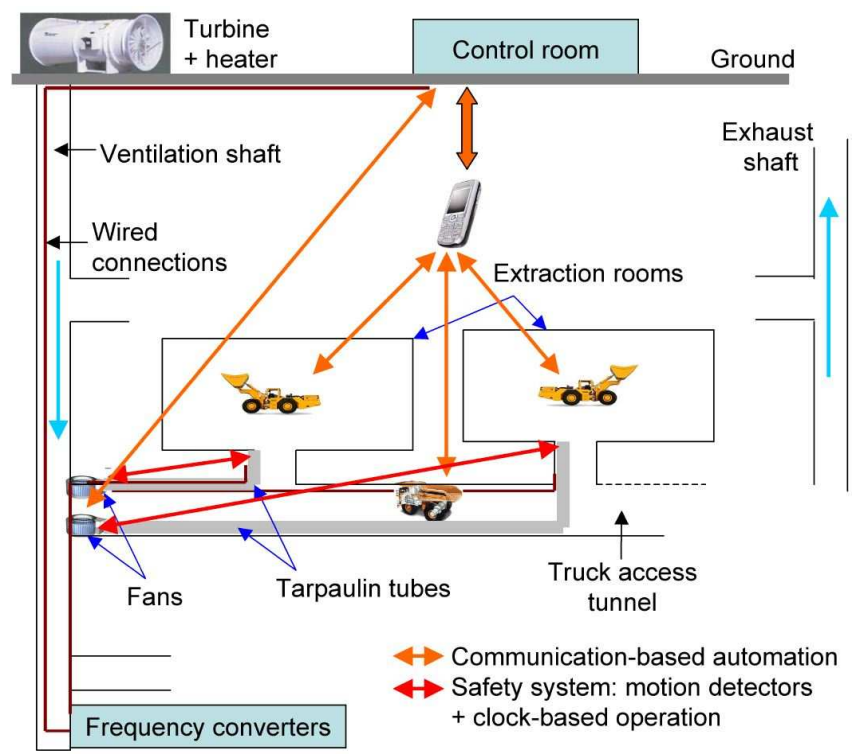

Figure 1. Mine ventilation process and current automation.

winter time at least) to avoid freezing in the upper part of the shaft and air cooling devices are used at high depths (more than 1000 meters) to compensate the geothermal effect (the temperature increases by $1^{\circ} \mathrm{C}$ every $30 \mathrm{~m}$ as we go down). We will refer to this part of the system as the primary system. From the ventilation shaft, fans located at each extraction level pump fresh air to the extraction rooms via tarpaulin tubes: this is the secondary system. Bad quality air naturally flows because of the pressure gradient from the extraction rooms back into the decline and to the exhaust ventilation shaft (similar but separate from the primary ventilation shaft).

The distinction between primary system and secondary system will be used in the following sections to split the control problems. In fact, the primary system typically has a simpler and more stable geometry while the secondary system is strongly varying in geometry (rooms are blasted every day), characteristics (tarpaulin tube length and shape) and disturbances (trucks) even within the same mine. Computational Fluid Dynamics (CFD) models, such as the one presented in [19] can then be envisioned for the primary system while grey-box identification or global models focused on the main dynamics should be preferred for the secondary one.

2.1.1. Today's automation architecture. In the current ventilation regulation architecture, the turbine is operated based on the flow pressure gradient and the heating depends on the external temperature. The system of fans is controlled according to the demand of airflow in different parts of the mine thanks to frequency converters, as presented in Fig. 1. This airflow demand is simply activated on messages sent by human operators to the control room using walkie-talkies, indicating their position and which fan needs to be set to its maximum speed (no automatic control, but maximum ventilation power during ore extraction). Both turbine 
and fans are at least operated at low speed for safety reasons. There may also be an additional safety system that triggers the fan high-speed operation based on a motion detector placed at the entrance of the extraction rooms. The current control architecture is then characterized by the absence of any true sensing of air quality and of real-time feedback loops in the secondary system.

2.1.2. Definition of control objectives. The main objective of the mining control system is to provide the extraction rooms with good air quality, thus referring to a ventilation control. We specify the objective as the control of air quality $\left(\mathrm{O}_{2}, \mathrm{NO}_{x}\right.$ and/or $\left.\mathrm{CO}_{x}\right)$ in the extraction rooms at different levels. Considering the distinction between the primary and secondary systems as defined above, it is then suitable to fulfill a cascade control configuration with the following two objectives:

1. regulate the turbine and heater based on physical measurements within the vertical shaft to provide a suitable airflow pressure at the location of the fans;

2. regulate the ventilation fans based on chemical sensors to ensure air quality in extraction rooms.

An additional system objective is to obtain safety through wireless networking (e.g. support of personal communication and localization). It should be noted that todays control architecture does not enable the fulfillment of these objectives, due to the lack of automatic control and sensing capabilities. Instead the wireless control architecture that is proposed in the following strives for fulfilling all the objectives listed above. Moreover, due to the advanced features of this architecture, additional control objectives can be defined, e.g. in terms of personal safety for mining personnel (localization, real-time process monitoring, etc.).

\subsection{Proposed wireless control architecture}

The proposed automation architecture is depicted in Fig. 2, where we introduced networked sensors in the vertical ventilation shaft, in access tunnels and in the extraction rooms. The sensors placed in the vertical shaft and in the access tunnels can make use of the existing wired connections, while those in the extraction rooms must be wireless, due to the blasting activities. The exchange of sensor measurements and control signals can then be carried through a mix of wired and wireless links.

2.2.1. Hybrid wired-wireless communication. Since the whole system setup foresees the presence of some cabling, for power delivery to fans along the primary system and for connecting entrance detectors in rooms in the secondary system, it seems interesting to investigate the exploitation of power line communication (PLC) devices. The main idea is to provide a wired backbone along the power line already present in the system, while resorting to a proper WSN deployment for air sensing within the extraction rooms. In this setup a proper PLC/WSN gateway node should be deployed at the interface between the area and the wired backbone.

This basic setup can be further enhanced by exploiting the fact that the working environment is typically populated by several mobile entities like trucks and humans. Such entities (let them be equipped with one or more sensor nodes) could be useful to improve the quality of monitoring by increasing the spatial density of measurements. While the mobile entities are 


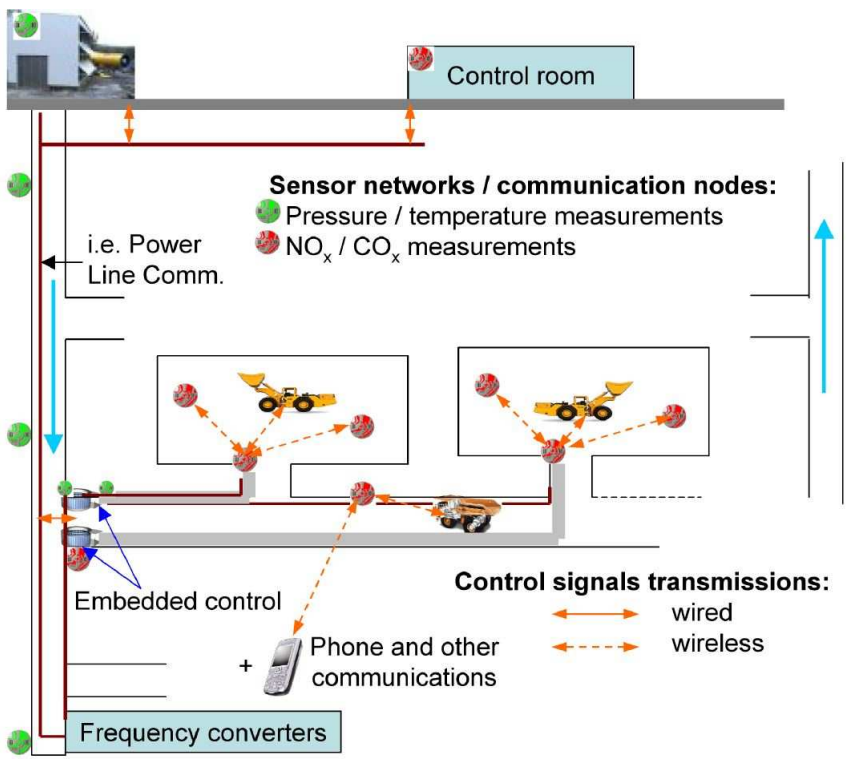

Figure 2. Proposed automation architecture.

a potential benefit from an energy point of view (as the wireless nodes can resort to classical battery recharge mechanisms), their deployment poses additional challenges as it induces an increased topology variability in space and time. Along that line, an interesting evolution is concerned with hand-held and/or on truck mobile nodes acting as cluster-heads and in turn interconnected (e.g. through an IEEE 802.11 wireless interface) to build a dynamic wireless backbone. This would help to make energy constraints on some lower tier wireless sensor nodes less stringent, while providing a larger bandwidth support for introducing advanced services, like, for example, an IP-based (peer-to-peer) voice service [7].

In the next sections, we suppose that such a network is deployed and ensures the necessary communication capabilities for distributed sensing and actuation.

\subsubsection{Decentralized control and fans regulation. Two control architectures can be considered:}

1. a centralized strategy, where all the sensor signals are transmitted to the control room, where the control algorithms are run. This strategy aims to global optimization of the energy consumption, at the price of a higher communication load due to the transmission of control signals between the surface and the fans;

2. a decentralized approach, where some intelligence is embedded at the locations of the fans and can adjust their regulation directly based on the chemical sensors measurements. This regulation is structurally sub-optimal but should be preferred as a first approach for its simplicity and the limited communication constraints.

Note that a control algorithm embedded at a fans location has to be relatively simple, because of the limited computation capabilities, while we can develop more complex control strategies for the algorithms run in the control room. 


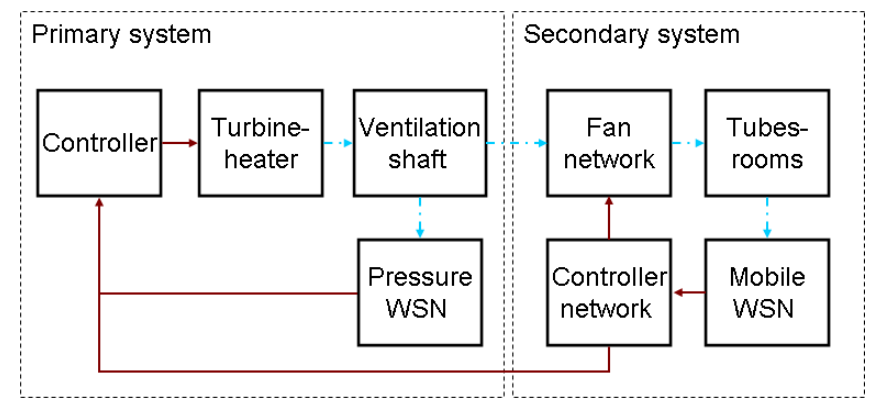

Figure 3. Description of the control setup, including communications $(-)$ and airflows $(-\cdot-)$.

The ventilation system can be described in two parts: one fixed installation, which is the primary air supply from the ground via a vertical shaft, and the secondary system, a mobile network of fans. This architecture can be abstracted in the block diagram description proposed in Fig. 3, where both the physical (airflow) and communication (wired and wireless) interconnections are modeled. This description includes the existing systems as well as key elements of the advanced technological solutions presented in this paper.

Considering the fact that a feedback controller (using the pressure gradient) is already available on the primary system, we will focus on proposing feedback control strategies that can be implemented on the secondary system. We suppose that the primary system is regulated so that a fixed pressure is available for the fans and will detail in the next sections the main dynamics of the secondary system, the importance of the WSN in the controlled dynamics and two possible model-based control strategies.

\section{EXTRACTION ROOMS AIR QUALITY MODEL}

Clearly, model-based control strategies have a significant advantage to ensure optimized performances and handle classical control problems, such as actuation and communication constraints, disturbances rejection and energy consumption minimization. In order to provide a global control strategy for a large-scale interconnected system, we first present a simplified fluid model that makes use of the available distributed measurements. The aerodynamics in the room environment is mainly set by the gas buoyancy and resulting stratification. In the extraction rooms, the pollutant sources from trucks can be considered as forced plumes, the incoming fresh air as a jet and the gas in the room as a stratified flow. Specific simplified fluid models have been proposed to represent such flows in $[20,21]$, which gave rise to an active research field in fluid mechanics. The development of Intelligent Buildings automation, and particularly Underfloor Air Distribution systems [22], renewed the interest in these models, due to the associated potential costs and energy savings (buildings air conditioning currently represents $10 \%$ of all energy use in the US).

We consider the secondary system, detailed in Fig. 4. Distributed wireless sensors provide chemical measurements at different locations in the extraction room. We consider that the sensors signals are carried to the fans embedded control units over a wireless multi-hop network, 
to account for the fact that a wired network would be difficult to install and maintain in such environment. We suppose that there is no pollutant in the tarpaulin tube and that the flow in the room is incompressible.

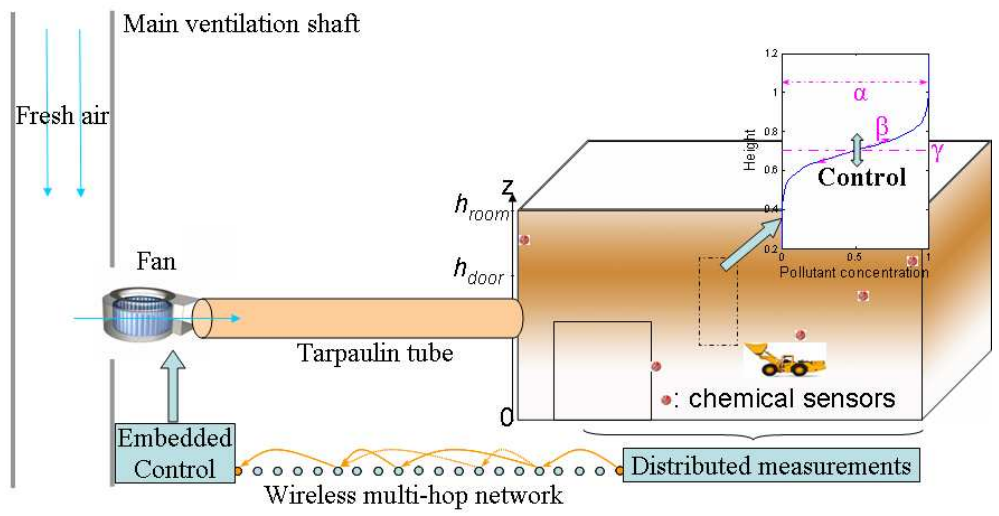

Figure 4. Stratification and sigmoid description in extraction rooms.

Most of the proposed dynamics rely on a non-dimensional modeling of the distributed phenomena. The two main advantages of this approach, classically used in industrial preliminary design, are the integration of as many physical properties as possible (avoid data mapping) and a reduced computation time (close to real-time, approximately 10 times slower in the worst cases). It then allows for predictive models on non-identified operating points with satisfying computation times.

\subsection{Time-delays}

Two different delays are involved in this model. The first one is a physical delay, associated with the airflow in the tarpaulin tube, between the fan and the extraction room (the time needed for a change of the flow speed due to a modification in the fan actuation to reach the extraction room). This airflow is considered inviscid and incompressible, and modeled as a time-varying delay $\tau_{\text {tarp }}(t)$. Indeed, for a 1-dimensional Poiseuille laminar flow and the previous hypotheses, the flow speed $u(x, t)$ and temperature $T_{\text {tarp }}(x, t)$ are obtained from Navier-Stokes equations (see, for example, [23] or similar textbooks for details) as:

$$
\frac{\partial}{\partial t}\left[\begin{array}{c}
u \\
T_{\text {tarp }}
\end{array}\right]+\left[\begin{array}{cc}
u & r \\
\gamma T_{\text {tarp }} & u
\end{array}\right] \frac{\partial}{\partial x}\left[\begin{array}{c}
u \\
T_{\text {tarp }}
\end{array}\right]=0,
$$

where $r=287.045 \mathrm{~J} / \mathrm{kg} . \mathrm{K}$ is the air gas constant and $\gamma=1.4$ is the ratio of specific heat coefficients. The characteristic velocities $v(x, t)$ of the traveling waves are then the solutions of:

$$
\operatorname{det}\left|\begin{array}{cc}
-v+u & r \\
\gamma T_{\text {tarp }} & -v+u
\end{array}\right|=0 \quad \Leftrightarrow \quad v_{1,2}(x, t)=u(x, t) \pm \sqrt{r \gamma T_{\text {tarp }}(x, t)} .
$$


We are interested in the down-flow time-delay, which is approximated by the previous equation as:

$$
\tau_{\text {tarp }}(t) \approx \frac{L_{t}}{\bar{u}(t)+\sqrt{r \gamma \bar{T}_{\text {tarp }}(t)}},
$$

where $L_{t}$ is the length of the tarpaulin tube, $\bar{u}(t)$ and $\bar{T}_{\text {tarp }}(t)$ are the space-averaged flow speed and temperature, respectively.

The second source of delay is due to the distributed measurements and wireless transmission between the extraction room and the fan. This delay is time-varying and denoted as $\tau_{w s n}(t)$, to indicate that it is related to Wireless Sensor Network (WSN) automation. We consider that a wireless multi-hop protocol is set, as detailed in [24], to minimize the energy consumption of the wireless nodes according to specific communication quality constraints. The associated end-to-end delay writes as:

$$
\tau_{w s n}(t)=h(t) F+\sum_{i=1}^{h(t)}\left(\alpha_{i}+\beta_{i}\right),
$$

where $h(t)$ is the time-varying number of hops, $F$ denotes the propagation and transmission delays, $i=1 \ldots h(t)$ indicates the transmission node considered, $\alpha_{i}$ is the time to wait before sending a data packet (typically a random variable) and $\beta_{i}$ is the time induced by an Automatic Repeat reQuest (ARQ) mechanism. For simulation purposes, we consider the experimental data presented in [25], scaled to represent a tunnel of length $L_{t}$ (initial measurements performed in a $10 \mathrm{~m}$ corridor, approximately). Note that packet losses (not detailed here) are associated with this model, as the number of packets losses is directly proportional to the number of hops.

\subsection{Energy losses in the tarpaulin tube}

The loss of airflow energy due to curves and length of the tube can be modeled using standard loss parameters as follows. The fan produces a pressure gradient that results in a flow speed $u_{f a n}$ at the entrance of the tarpaulin tube. This speed is then increased by the negative variation of pressure $\Delta H=P_{f a n}-P_{\text {room }}$ between the input and the output of the tube, and decreased by the energy losses (friction). The flow energy is partly dissipated along the tarpaulin tube (distributed losses $\xi_{d}$ ) and in the curves (concentrated losses $\xi_{c}$ ). Conservation of energy (Bernoulli's equation) implies that:

$$
\Delta H=\xi_{d}+\xi_{c}+\rho \frac{u_{\text {room }}^{2}}{2}-\rho \frac{u_{\text {fan }}^{2}}{2},
$$

where $\rho$ is air density and $u_{\text {room }}$ is the flow speed at the room side of the tube. We can express the distributed losses by $\xi_{d}=L_{t} \rho u_{\text {avg }}^{2} f /\left(2 D_{t}\right)$ (Darcy-Weisbach equation), where $D_{t}$ is the tube diameter, $f$ models friction losses, and $u_{\text {avg }}$ is the average velocity of the fluid in the tube. We take into account the concentrated losses introduced by the curves considering an effective length $L_{e}=\sigma L_{t}, \sigma>1$. The value of $\sigma$ depends on the curvature and diameter of the tube. Replacing $u_{\text {avg }}$ with $u_{f a n}$ (over-approximation of the losses), we have that $\xi_{d}+\xi_{c}=L_{e} \rho u_{f a n}^{2} f /\left(2 D_{t}\right)$. For the considered operating conditions, the flow is turbulent (i.e. the Reynolds number $R e$ is very high) and the energy losses are further simplified considering that $f$ can be expressed as [26]:

$$
f=\left(1.82 \log _{10} R e-1.64\right)^{-2} \Rightarrow \xi=\xi_{d}+\xi_{c}=\frac{1}{2} \frac{L_{e}}{D_{t}} \rho\left(\frac{u_{f a n}}{1.82 \log _{10} R e-1.64}\right)^{2} .
$$


By substituting (3) in (2) and solving for $u_{\text {room }}$ we obtain:

$$
u_{\text {room }}(t) \approx \eta \times u_{\text {fan }}\left(t-\tau_{\text {tarp }}\right), \quad \eta \doteq \sqrt{1-\frac{\sigma L_{t}}{D_{t}}\left(\frac{1}{1.82 \ln R e-1.64}\right)^{2}},
$$

where $\eta<1$ represents the energy losses associated with the tarpaulin tube and $R e \approx$ $67280 D_{t} \times u_{f a n}$ [4]. We supposed that $2 \Delta H /\left(\rho u_{f a n}^{2}\right)<<1$, which is a reasonable assumption associated with the incompressibility hypothesis in the tarpaulin tube.

\subsection{Concentration profiles}

The novel modeling approach presented in this section is primarily motivated by the shapes similarity of the experimental buoyancy profiles presented in [22] and related works. Indeed, fluid stratification (relative gravity) monitored for different inflow buoyancy, momentum and number of sources, always exhibits sigmoid-like profile. WSN measurements motivate further use of this property, as they can be easily associated with an appropriate estimation strategy to provide "on-line" shape monitoring. This extra simplification is particularly suitable to the proposed global approach. Establishing the control strategy on the shape properties also has the significant advantage that, compared with classical space discretization methods, the closed-loop performance is structurally robust with respect to the time-varying localization of the WSN measurements.

Note that, as the clean airflow and pollutants enter directly into the extraction rooms, we do not consider the physical transport phenomenon (or associated time-delay) in the rooms but only first order dynamics on mass conservation. This is consequently equivalent to a 0-D model of the extraction room. The associated model uncertainties can be considered as acceptable since the distributed measurements provided by the WSN allow for an online model adjustment using classical estimation methods.

The pollutants $\left(\mathrm{CO}_{x}, \mathrm{CH}_{4}, \mathrm{SO}_{2}\right.$ and $\mathrm{N}_{x} \mathrm{O}_{x}$ are classically associated with the trucks engines) volume concentration profiles $c_{j}(z, t)$, where $z \in\left[0, h_{\text {room }}\right]$ is the height in the extraction room, $h_{\text {room }}$ is the room height and $j$ indicates the pollutant considered, is approximated by the sigmoid distribution:

$$
c_{j}(z, t)=\frac{\alpha_{j}(t)}{1+e^{-\beta_{j}(t)\left(z-\gamma_{j}(t) h_{\text {room }}\right)}},
$$

where $\alpha_{j}(t)$ is the amplitude, $\beta_{j}(t)$ is the dilatation and $\gamma_{j}(t)$ is the inflection point of the distribution. Note that any function can be approximated with the desired precision level by a sum of such sigmoid functions: we suppose here that one curve is sufficiently accurate for feedback control purposes and that the induced error is negligible in comparison with the system uncertainties. This simplified modeling approach was proposed for distributed systems involving smooth energy transport phenomena in [27] for the modeling of temperature profiles in Tokamak plasmas and validated with experimental results.

In our case, the real-time measurements provided by the WSN can be used to estimate the shape parameters $\left\{\alpha_{j}, \beta_{j}, \gamma_{j}\right\}$ thanks to classical recursive identification or Kalman filtering techniques [28]. To ensure the system identifiability, we have to suppose that measurements are available at three different heights for each regulated pollutant. We can consider that a node contains all the necessary chemical sensors, which simplifies the previous hypothesis to the need of three wireless sensor nodes located at different heights. Note that the heightdependency of the concentration distribution allows for a direct use of all the available 
information at a given sampling time if we have more than three nodes (e.g. we can easily substitute the information provided by a sensor by the one provided by another if we keep $z$ as a free variable in the estimation algorithm). The model accuracy then directly depends on the measurements frequency, the number of nodes and the precise knowledge of the nodes localization (e.g. achieved thanks to ultra-wide band techniques for very accurate positioning in harsh environments such as underground mines, as proposed in $[29,30])$.

As the aim of this paper is to investigate the impact of the main dynamics on the feedback regulation and since no experimental data is available for the moment, we only describe the impact of the mass conservation (flow continuity property). This ensures that the proposed model is consistent with the global physics of the system and can be used in a predictive mode. The shape parameters $\left\{\alpha_{j}, \beta_{j}, \gamma_{j}\right\}$ can also be related to the global parameters (room temperature and pressure, number of trucks and engines power, etc.) with an appropriate identification method. The pollutant mass $m_{j}(t)$ in the room is obtained from the concentration distribution thanks to the relationship:

$$
m_{j}(t)=S_{\text {room }} \int_{0}^{h_{\text {room }}} c_{j}(z, t) d z \approx S_{\text {room }}\left[\int_{0}^{h_{\text {door }}} c_{j}(z, t) d z+\alpha_{j}(t) \Delta h\right],
$$

where $S_{\text {room }}$ is the room surface, $h_{\text {door }}$ is the door height and $\Delta h=h_{\text {room }}-h_{\text {door }}$. The last equality is established by supposing that the air above $h_{\text {door }}$ is saturated with pollutants, as there is no exhaust in this region. Furthermore, we are interested in a detailed model below the room entrance level, as it corresponds to the breathing and engine levels, and can consider the term $\alpha_{j}(t) \Delta h$ as an averaging of what happens above. This hypothesis is reasonable in mining ventilation applications as, typically, the ceiling is blasted and the engines/humans enter the room only to work on the ore at the ground level. The pollutant dynamics is set thanks to the mass conservation law:

$$
\dot{m}_{j}(t)=\dot{m}_{j, \text { in }}(t)-\dot{m}_{j, \text { out }}(t)-\dot{m}_{j, \text { chem }}(t),
$$

where $\dot{m}_{j, i n}$ is the incoming pollutant mass rate due to the engines and humans, given by appropriate specifications, and $\dot{m}_{j, c h e m}$ is the mass variation due to chemical reactions between components $j$ and $k$ at a rate $\nu_{j k}=-\nu_{k j}$. The mass conservation equation (4) sets the shape parameters dynamics with:

$$
\begin{aligned}
\dot{m}_{j}(t) & \approx S_{\text {room }}\left[\int_{0}^{h_{\text {door }}} \dot{c}_{j}(z, t) d z+\dot{\alpha}_{j}(t) \Delta h\right], \\
\dot{m}_{j, \text { out }}(t) & \approx \frac{Q_{\text {out }}}{h_{\text {door }}} \int_{0}^{h_{\text {door }}} c_{j}(z, t) d z, \\
\dot{m}_{j, \text { chem }}(t) & =S_{\text {room }}\left[\int_{0}^{h_{\text {door }}} \nu_{j k} c_{j}(z, t) c_{k}(z, t) d z+\nu_{j k} \alpha_{j}(t) \alpha_{k}(t) \Delta h\right],
\end{aligned}
$$

where $Q_{\text {out }}=S_{t} \eta u_{\text {fan }}\left(t-\tau_{\text {tarp }}\right)$ is the volume rate of flow leaving the room (equal to the one entering the room, from the incompressibility hypothesis), while $S_{t}$ is the cross-section of the tarpaulin tube. The dynamics of the mass transformed by chemical reactions $\dot{m}_{j, c h e m}(t)$ is set using the classical equation $\dot{m}_{j, c h e m}(t)=\nu_{j k} m_{j} m_{k}$, applied to our room division (above and below $\left.h_{\text {door }}\right)$ and expressed in terms of the concentration. This model ensures that the transformed mass is conserved $\left(\dot{m}_{j, \text { chem }}+\dot{m}_{k, \text { chem }}=0\right)$. 
The variable-step integration method described in [27] (discretization of the integral term, that is expressed as a constant matrix) is now introduced to obtain a differential equation representation of the distributed model:

$$
E_{j}\left[\begin{array}{c}
\dot{\alpha}_{j}(t) \\
\dot{\beta}_{j}(t) \\
\dot{\gamma}_{j}(t)
\end{array}\right]=\dot{m}_{j, i n}(t)-B_{j} u_{f a n}\left(t-\tau_{\text {tarp }}\right)-D_{j k},
$$

with:

$$
\begin{aligned}
E_{j} & \doteq S_{\text {room }}\left(V_{\text {int }}\left[\begin{array}{ccc}
\vdots & \vdots & \vdots \\
\frac{\partial \dot{C}_{j, i}}{\partial \alpha_{j}} & \frac{\partial C_{j, i}}{\partial \beta_{j}} & \frac{\partial C_{j, i}}{\partial \gamma_{j}} \\
\vdots & \vdots & \vdots
\end{array}\right]+\left[\begin{array}{c}
\Delta h \\
0 \\
0
\end{array}\right]^{T}\right], \\
B_{j} & \doteq \frac{1}{h_{\text {door }}} V_{\text {int }}\left[\begin{array}{c}
\vdots \\
C_{j, i} \\
\vdots
\end{array}\right] \times S_{t} \eta, \quad D_{j k}=S_{\text {room }} \nu_{j k, i}\left[V_{i n t}\left[\begin{array}{c}
\vdots \\
C_{j, i} C_{k, i} \\
\vdots
\end{array}\right]+\alpha_{j} \alpha_{k} \Delta h\right],
\end{aligned}
$$

where $i=1 \ldots N, V_{\text {int }}=M_{\text {int }}(N, 1: N), N$ is the number of discretization points, $M_{\text {int }}$ is the integration matrix and $C_{j, i}=c_{j}\left(z_{i}, t\right)$. The system dynamics is finally represented as a set of delay differential equations of the form:

$$
\begin{aligned}
{\left[\begin{array}{c}
\dot{\alpha}_{j}(t) \\
\dot{\beta}_{j}(t) \\
\dot{\gamma}_{j}(t)
\end{array}\right] } & =E^{\dagger}\left[\dot{m}_{j, i n}(t)-B_{j} u_{\text {fan }}\left(t-\tau_{\text {tarp }}\right)-D_{j k}\right] \\
y_{j}(t) & =\frac{\alpha_{j}(t)}{1+e^{-\beta_{j}(t)\left(z_{r}-\gamma_{j}(t) h_{\text {room }}\right)}},
\end{aligned}
$$

where $\dagger$ denotes the Moore-Penrose inverse and $y_{j}(t)$ is the concentration of gas $j$ at the reference height $z_{r}$.

\subsection{Locally regulated fan}

We used a three-phase asynchronous motor model with the fan as load to determine the fan power consumption. The working principle can be found in [31] or in similar text books. We report here the main principles. Let $n_{2}$ be the speed of the motor, then:

$$
n_{2}=\frac{60 f_{1}}{p}(1-s)
$$

where $f_{1}$ is the supply frequency and $p$ the number of the poles. The slip factor of the motor is defined as $s \doteq\left(n_{1}-n_{2}\right) / n_{1}$, where $n_{1}$ is the speed of the rotating magnetic field. A local feedback control loop is set by varying the supply frequency while maintaining a constant voltage-frequency ratio. This kind of control is called scalar $\mathrm{V} / \mathrm{Hz}$ control [31]. The parameters of the motor and the fan, along with their numerical values are shown in Tab. I.

\subsection{Illustrative test case.}

To illustrate the previous results, we consider an extraction room with dimensions $h_{\text {room }}=$ $9 \mathrm{~m}, S_{\text {room }}=200 \mathrm{~m}^{2}$ and $h_{\text {door }}=5 \mathrm{~m}$, and wish to regulate the air quality at $z_{r}=3 \mathrm{~m}$. 


\begin{tabular}{ll|ll}
\hline \multicolumn{2}{l}{ Motor and Fan Characteristics } & \multicolumn{3}{l}{} \\
\hline Nominal Power & $75 \mathrm{KW}$ & Nominal RMS Voltage & $400 \mathrm{~V}$ \\
Nominal Current & $155 \mathrm{~A}$ & Nominal Frequency & $50 \mathrm{~Hz}$ \\
Nominal Frequency & $50 \mathrm{~Hz}$ & Nominal Speed & $1484 \mathrm{RPM}$ \\
Nominal Speed & $\sim 155,32 \mathrm{rad} / \mathrm{sec}$ & Nominal Torque & $\sim 483 \mathrm{Nm}$ \\
Maximum Voltage & $\sim 565 \mathrm{~V}$ & Motor Friction & 0.03914 \\
Rotor Resistance & $20.92 \mathrm{~m} \Omega$ & Rotor leakage Inductance & $0.335 \mathrm{mH}$ \\
Stator Resistance & $35.52 \mathrm{~m} \Omega$ & Stator leakage Inductance $0.335 \mathrm{mH}$ \\
Magnetizing Inductance & $0.0151 \mathrm{mH}$ & Base Frequency & $50 \mathrm{~Hz}$ \\
Number of poles & 4 & Motor Inertia & $1.21 \mathrm{Kg} / \mathrm{m}^{2}$ \\
Motor Friction & 0.03914 & Base Speed & $314 \mathrm{rad} / \mathrm{sec}$ \\
Stator Impedance & $105 \mathrm{~m} \Omega$ & Rotor Impedance & $105 \mathrm{~m} \Omega$ \\
Magnetizing Impedance & $4.74 \Omega$ & Fan Friction & 0.13 \\
Load Inertia & $4 \mathrm{Kg} / \mathrm{m}^{2}$ & &
\end{tabular}

Table I. Motor and Fan Characteristics

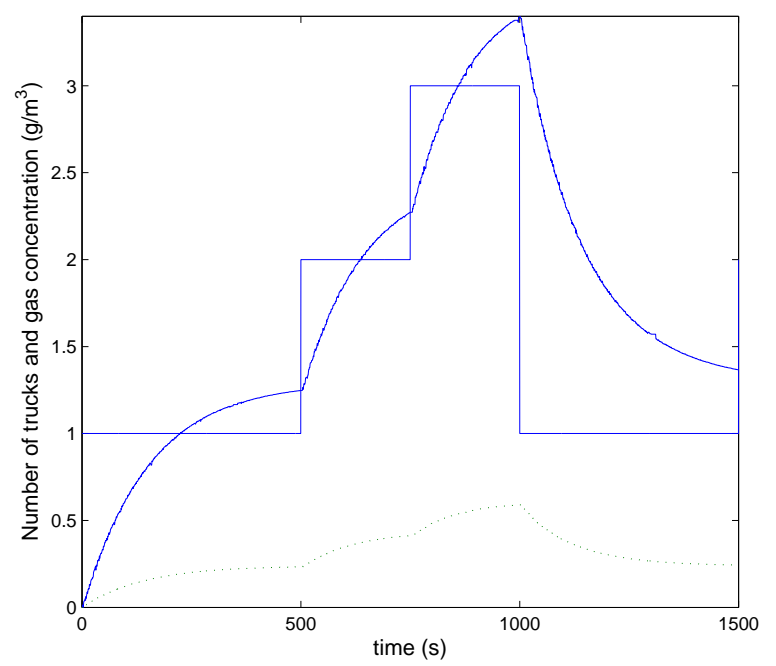

Figure 5. CO (- -) and NO (-) concentrations in the extraction room.

The system (room and tarpaulin tube) temperature is $298 \mathrm{~K}$ and its pressure is $1 \mathrm{~atm}$. The tube parameters are $D_{t}=1.2 \mathrm{~m}, L_{t}=300 \mathrm{~m}$ and $\sigma=1.66$. We consider the regulation of two toxic gases emitted by the trucks, $C O$ and $N O$, with an atmospheric concentration chosen arbitrarily as $10^{-6} \mathrm{Kg} / \mathrm{m}^{3}$ (trace gases). The engines emit approximately $23.5 \mathrm{~g} / \mathrm{s}$ of $\mathrm{NO}$ and $4.6 \mathrm{~g} / \mathrm{s}$ of $C O$ (inferred from [32]), and we consider the safety thresholds $\overline{N O}=5.9 \mathrm{~g} / \mathrm{m}^{3}$ and $\overline{C O}=0.234 \mathrm{~g} / \mathrm{m}^{3}$ (calculated from [4] for sea level conditions). The network delays and 
packet losses are obtained by scaling the measurements presented in [25] to the system scale: this results in communication delays between 1 and $5 s$ and packet losses ranging from 10 to $40 \%$. The time evolution of the concentrations at $z_{r}$ is presented in Fig. 5, for a fan flow speed $u_{f a n}=10 \mathrm{~m} / \mathrm{s}$ and 1 to 3 trucks working in the room. In this scenario, $\overline{C O}$ is clearly the limiting factor, but the relative importance of the components strongly depends on the chemical reactions rate, which is chosen arbitrarily.

Remark 1. The proposed non-dimensional dynamics has been constructed based on the fundamental conservation laws (flow equation for the physical delay, mass conservation in the room), engineering relationships (Darcy-Weisbach equation and associated energy losses computation, fan model) and experimental measurements (scaled WSN delays and packet losses). This part of the model is then fairly accurate to investigate the main dynamics, compare the time constants and test different control strategies. The concentration profiles estimation with sigmoid curves constrained by first order dynamics (mass conservation) is sufficient for control design analysis at this point, as the main time constant and some nonlinearities are roughly depicted. Specific care has to be taken in its real-time implementation as it strongly relies on the distributed measurements provided by the WSN and on their localization.

The communication constraints, as time-delays and packet losses, are of secondary importance here due to the slow behavior of the concentration profiles evolution. These constraints will only be considered as light constraints in the control schemes. This reduces the inclusion of WSN into the solution to a conceptual level, as an automation solution that provides the necessary distributed measurements. Future works focused on the communication aspects may consider more specifically the impact of communication outages or the design of "sleep and wake" sensors policy according to the pollutants dynamics (i.e. maximizing the sleeping time to minimize the sensors energy consumption without degrading the closed-loop performances).

\section{CONTINUOUS APPROACH: NONLINEAR MODEL PREDICTIVE CONTROL}

The control objective is to minimize the fan energy consumption while ensuring an acceptable air quality in the extraction room. Due to the height-dependent model proposed in the previous section, the air quality objective can be rephrased as guaranteeing a maximum pollutant concentration at a given height $z_{r}$ :

$$
\max _{\forall j} y_{j}(t) \leq \bar{y}_{j}
$$

where $\bar{y}_{j}$ is the threshold value on pollutant $j\left(C O_{x}\right.$ or $N O_{x}$ for example). The reference $z_{r}$ corresponds to the highest height where the air quality has to be guaranteed and around which the sensors should be placed. Communication constraints, such as delays, timeout, packet losses and bandwidth limitations are also taken into account in the optimization algorithm.

\subsection{Receding Horizon Control}

Predictive control is a model-based design technique [33, 34], which basic principles are illustrated in Fig. 6. It is based on the on-line solution of successive optimization problems. The idea is to solve a scheduling optimization problem at time $k T$, ( $T$ : sampling time) from a 
prediction model, based on future desired outputs $y_{j, d e s}$ and prediction of future disturbances $\hat{\dot{m}}_{j, i n}$ (pollutant sources from trucks in this case), to apply the first control values to the system, update the prediction model according to the available measurements, and repeat the whole procedure at time $(k+1) T$. The desired outputs are chosen as close as possible to the maximum admissible pollutants concentrations, possibly with a safety margin, in order to minimize the fan energy consumption. The receding horizon controller then has to achieve the desired trajectory while respecting the concentration thresholds, which act as output boundary constraints.

The scheduling algorithm can be stated as:

$$
\min _{u_{i}, i \in\left\{1, \ldots, N_{u}\right\}} \int_{k T}^{k T+N}\left[\sum_{j}\left(\hat{y}_{j}(\tau)-y_{j, d e s}(\tau)\right)^{2}+\lambda u_{f a n}^{2}(\tau)\right] d \tau,
$$

where $u_{f a n}(\tau)=u_{i}$ if $\tau \in\left[k T+(i-1) N / N_{u}, k T+i N / N_{u}\right]$, and $\hat{y}_{j}$ is the prediction of future outputs, computed from the simulation of a prediction model. For the prediction model, predicted values of disturbances $\hat{\dot{m}}_{j, i n}$ are considered. The tuning parameters of the control law

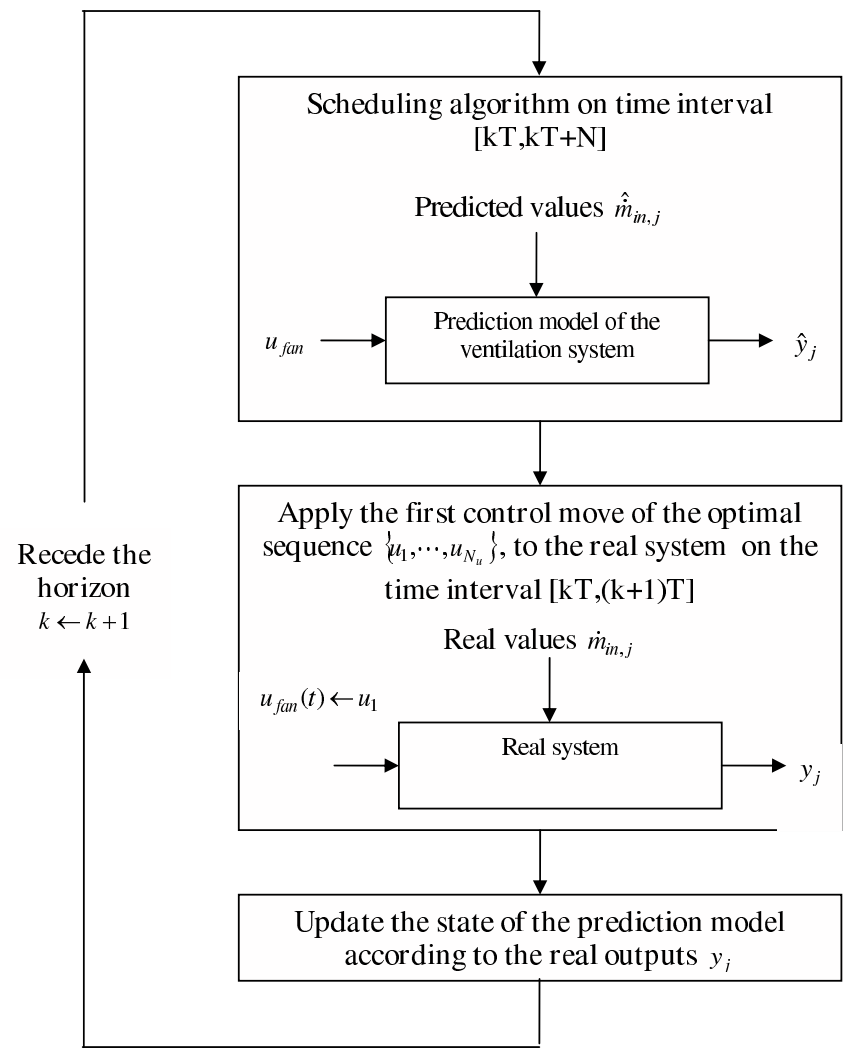

Figure 6. Receding horizon control synopsis.

are $N, N_{u}$ and $\lambda$. Here, $N$ represents the prediction horizon length. It should be chosen large 
enough to provide information about the transient behavior. Next, $N_{u}$ represents the number of degrees of freedom in the control action during the prediction horizon. A trade-off has to be found between the increased precision (large number of degrees of freedom) and the consequent augmented complexity of the optimal control problem to be solved at each sampling period. Finally, $\lambda$ is the weighting factor between the control effort and the disturbance rejection performances.

\subsection{Implementation issues}

In this paper the "real system" will be the reference model which has been defined in section 3. However, for a first approach, the following assumption is used for this section. The update of the states of the prediction model supposes that an estimation of the real system states is available from the signals of the wireless network. This part is beyond the scope of this paper, and in the following it is considered that the state of the system is available for control design purpose.

Being a real-time optimization procedure, the main implementation problems are related to the speed of convergence of the scheduling algorithm. This implies that the prediction model has to be simpler than the reference model. A trade off has to be made between its complexity and its representativeness. For the prediction model, the time varying delay $\tau_{\text {tarp }}(t)$ is chosen as a constant equal to the maximal time delay which may occur in the real system. This choice is the most robust one as it can only lead to a more reactive control law. The pollutant sources act as disturbances and are taken as the mean value of the sources due to the trucks in the prediction model. Any available information about the disturbances can be included in the prediction model.

The second important issue is the feasibility of the successive optimization procedure, as the scheduling algorithm has to be solved with the constraints defined in section 4.1. If the problem appears to be unfeasible, than one has to define alternative strategies. This point will be discussed in section 6.4 .

\subsection{Numerical result}

We focus on the constrained case and consider two pollutants, namely $C O_{x}$ and $N O_{x}$. The thresholds are defined as:

$$
\begin{gathered}
y_{C O_{x}}(t) \leq \bar{y}_{C O_{x}}=0.000234 \mathrm{~kg} \cdot \mathrm{m}^{3} \\
y_{N O_{x}}(t) \leq \bar{y}_{N O_{x}}=0.0059 \mathrm{~kg} \cdot \mathrm{m}^{3}
\end{gathered}
$$

The desired concentrations are set at $90 \%$ of the thresholds:

$$
\begin{aligned}
& y_{C O_{x}, \text { des }}=0.9 * \bar{y}_{C O_{x}} \\
& y_{N O_{x}, \text { des }}=0.9 * \bar{y}_{N O_{x}}
\end{aligned}
$$

The pollutant sources are set according to the scenario depicted in Fig. 5. For the prediction model, we consider a number of trucks equal to 2 , which is a mean value.

4.3.1. Reference case. The tuning parameters of the control law are:

- $N$ : prediction horizon of the model;

- $N_{u}$ : number of degrees of freedom for the control policy; 

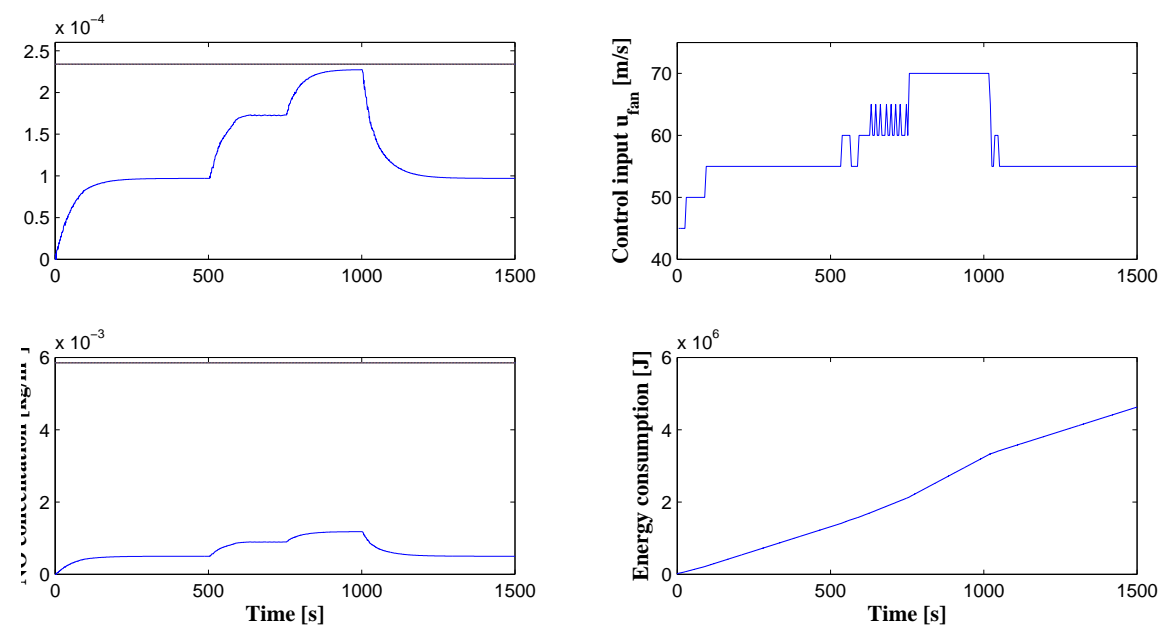

Figure 7. Regulation results for the reference case $\left(N=100 s, N_{u}=1, \lambda=10^{-7}\right)$.

- $\lambda$ : weighting factor between the controlled input consumption objective and the regulation objective.

The reference case is $N=100, N_{u}=1$ and $\lambda=10^{-7}$. The corresponding results are presented in Fig. 7, where the pollutants levels, control input and energy consumption of the electric motor of the fan are given. This regulation is satisfactory, with a smooth control action verifying the constraints over the whole time interval. As previously mentioned, the fact that a single actuator controls both pollutant concentrations $\left(N O_{x}\right.$ and $\left.C O_{x}\right)$ makes the control law to take into account the hardest constraint $\left(\mathrm{CO}_{x}\right)$.

The computation time for the simulation of $1500 \mathrm{~s}$ (real-time) is 10 minutes with Matlab ${ }^{T M}$ 2007a on a Pentium $I V^{T M}, 2.80 G H z$. The proposed regulation can then be performed in real-time, provided that sufficient computation capabilities are available. The influence of the tuning parameters will now be detailed.

4.3.2. Influence of $\lambda$. The reference case is modified by setting $\lambda=10^{-5}$ and the corresponding results are presented in Fig. 8. The fan control signal $u_{\text {fan }}(t)$ illustrates the fact that increasing $\lambda$ decreases the control inputs, as forecasted by the optimization problem formulation. Consequently, it also leads to a higher level of pollutant, but this level still satisfies the constraints.

4.3.3. Influence of $N$. The reference case is now changed by setting $N=50$. The corresponding results are given in Fig. 9. The control law is now more reactive, as the decisions of the controller related to the constraints activation are taken later than in the reference case.

4.3.4. Influence of $N_{u}$. Finally, the influence of $N_{u}$ is studied by testing the reference case with $N_{u}=2$, as illustrated in Fig. 10. The corresponding control law appears to be smoother, 

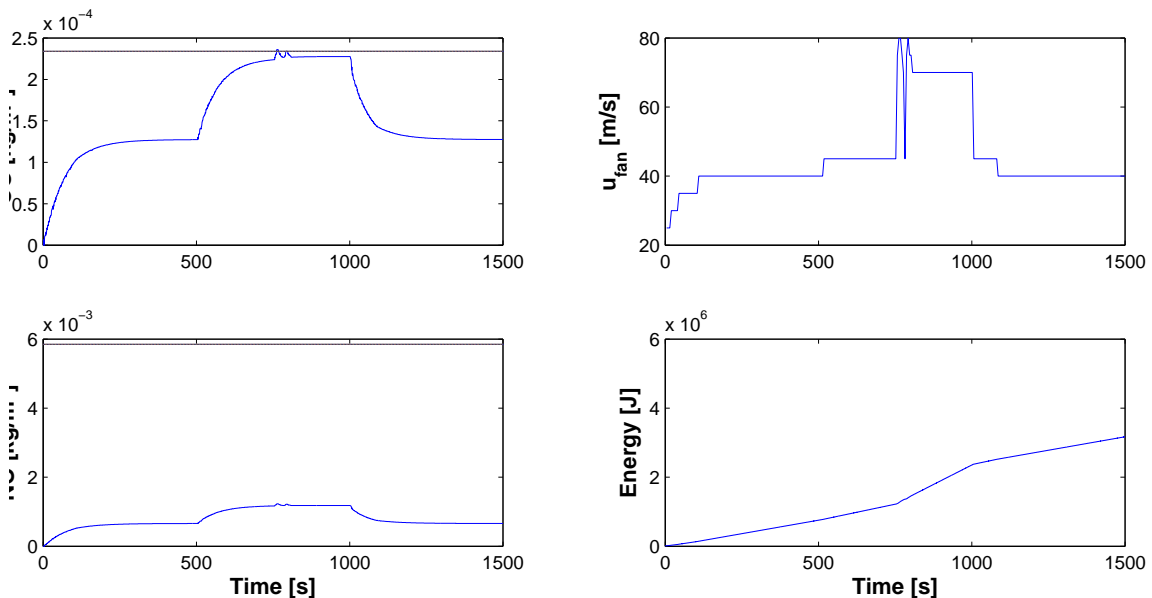

Figure 8. Influence of $\lambda$ on the reference case $\left(N=100 s, N_{u}=1, \lambda=10^{-5}\right)$.
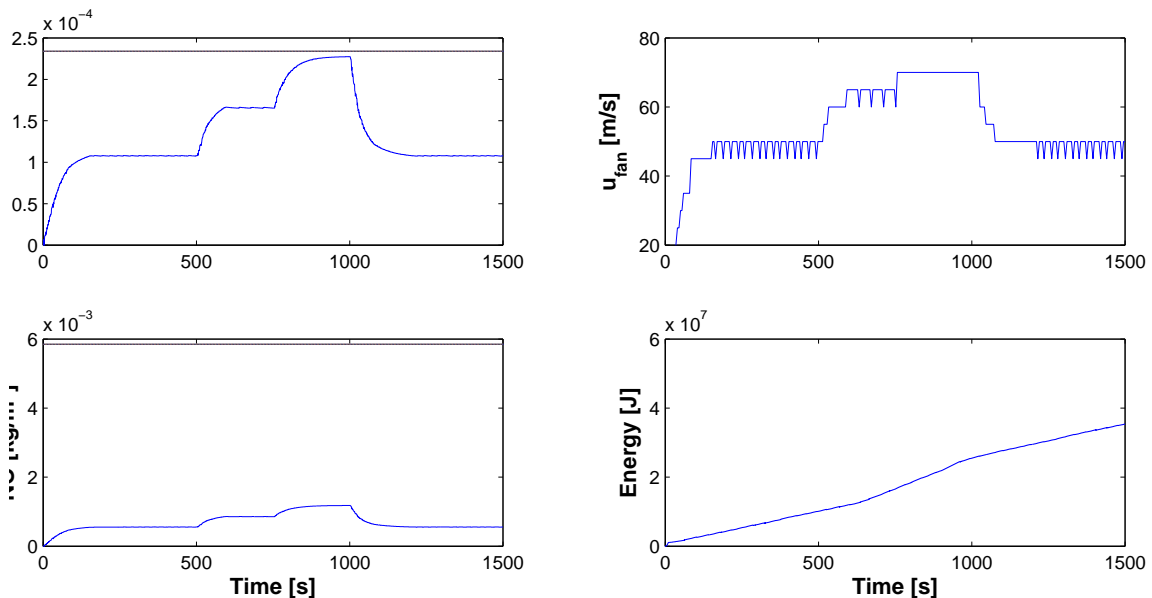

Figure 9. Influence of $N$ on the reference case $\left(N=50 s, N_{u}=1, \lambda=10^{-7}\right)$.

with very satisfactory regulation results. However, the computation time is increased to 45 minutes (4.5 times longer than the $N_{u}=1$ case). Remember that $N_{u}$ is the number of optimization variables, and thus is directly linked to the computation complexity.

Remark 2. The receding horizon control strategy has proved to be particularly efficient to achieve optimal performances on the nonlinear system considered. Communication constraints and model uncertainties are easily taken into account and the trade-off between regulation 

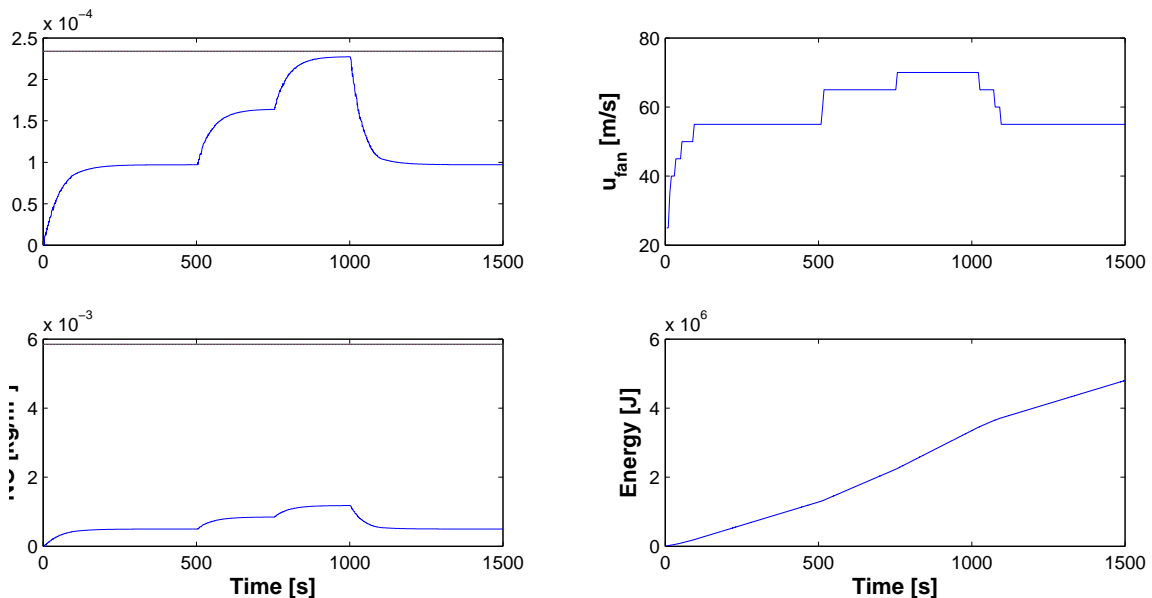

Figure 10. Influence of $N_{u}$ on the reference case $\left(N=100 s, N_{u}=2, \lambda=10^{-7}\right)$.

performance and energy consumption is transparent in the choice of the scalar parameter $\lambda$. The drawback is that the high computational demand associated with the optimization process may not be appropriate for embedded applications. This motivates a second control strategy, presented in the next section, where the algorithm simplicity is preferred to closedloop performance.

\section{HYBRID APPROACH: SAFETY CONTROL STRATEGY}

The control strategy proposed in this section is focused on the safety of the closed loop system. The secondary ventilation system is modeled conservatively, e.g. using worst case approximations, and we use a simple threshold control algorithm. We model the closed loop system using the hybrid systems mathematical framework: this allows to take into account the effects of time-delays, transmission errors and safety constraints. Thus, we use abstraction techniques and tools developed in previous works [35, 36] to verify safety (with respect to gas concentrations) and temporal properties of the system, with a required precision. Since we use a conservative approximation of the original system, if the safety verification algorithm states that the abstract system is safe then the original system is also safe.

\subsection{Hybrid model}

In Section 3.3 we derived the dynamics of the time-dependant shape parameters $\alpha(t), \beta(t)$ and $\gamma(t)$ in (5) and used them to compute the concentration of gas $j$ at the reference height $z_{r}(6)$. Since such model can not be used for the hybrid control approach proposed in this section, we derive approximated (conservative) dynamics for the gas concentration average. We are 
interested here in the dynamics of the height-averaged concentration:

$$
C_{j}(h, t) \doteq \frac{1}{h} \int_{0}^{h} c_{j}(z, t) d z .
$$

The pollutant dynamics is simplified by omitting the chemical reactions in the mass conservation law:

$$
\dot{m}_{j}(t)=\dot{m}_{j, \text { in }}(t)-\dot{m}_{j, \text { out }}(t)=\dot{m}_{j, \text { in }}(t)+u_{\text {in }}(t) S_{t} c_{a t m}-u_{\text {out }}(t) S_{\text {out }} C_{j}\left(h_{\text {out }}, t\right),
$$

where $c_{j, a t m}$ is the gas concentration in the atmosphere, $S_{\text {out }}$ is the section of the extraction room output, $S_{t}$ is the section of the tarpaulin tube, and $u_{i n}(t), u_{\text {out }}(t)$ are respectively the input and output airflow velocities. Dividing (8) by the room volume $V_{R}$, we obtain the concentration dynamics:

$$
\dot{C}_{j}\left(h_{\text {room }, t}\right)=\frac{\dot{m}_{j, i n}(t)}{V_{R}}+\frac{u_{i n}(t) S_{t} c_{j, a t m}}{V_{R}}-\frac{u_{\text {out }}(t) S_{\text {out }}}{V_{R}} C_{j}\left(h_{\text {out }}, t\right) .
$$

From the incompressibility hypothesis, (9) can be rewritten as:

$$
\dot{C}_{j}\left(h_{\text {room }}, t\right)=\frac{\dot{m}_{j, i n}(t)}{V_{R}}+\frac{u_{i n}(t) S_{t} c_{j, a t m}}{V_{R}}-\frac{u_{i n}(t) S_{t}}{V_{R}} C_{j}\left(h_{o u t}, t\right) .
$$

The next step is to simplify the profile description by a rough approximation of the shape parameters. We can use a hybrid representation, supposing that $\beta_{j}$ and $\gamma_{j}$ can only assume a finite number of constant values $\beta_{j}^{k}$ and $\gamma_{j}^{k}$, where $k$ is the number of trucks in the room. In other words, we do not consider the transient dynamics of these parameters when the number of trucks in the room changes. Note that $\beta_{j}^{k}$ and $\gamma_{j}^{k}$ should also depend on the current airflow velocity at the room entrance, but we suppose that the strongest contribution derives from the number of trucks in the extraction room. The dynamics of the average concentration in the room are calculated by expressing $\alpha_{j}(t)$ as a function of $C_{j}\left(h_{\text {room }}, t\right)$. To this aim, we substitute the sigmoid function in the integral (7) and obtain:

$$
\begin{aligned}
& C_{j}(h, t)=\frac{1}{h} \int_{0}^{h} \frac{\alpha_{j}(t)}{1+e^{-\beta_{j}^{k}\left(z-\gamma_{j}^{k} h_{\text {room }}\right)}} d z=\frac{\alpha_{j}(t)}{\beta_{j}^{k} h} \ln \left(\frac{e^{\beta_{j}^{k}\left(h-\gamma_{j}^{k} h_{\text {room }}\right)}+1}{e^{-\beta_{j}^{k} \gamma_{j}^{k} h_{\text {room }}}+1}\right), \\
& \Leftrightarrow \alpha_{j}(t)=\frac{\beta_{j}^{k} h_{\text {room }}}{\ln \left(\frac{e^{\beta_{j}^{k}\left(1-\gamma_{j}^{k}\right) h_{\text {room }}}+1}{e^{-\beta_{j}^{k} \gamma_{j}^{k} h_{\text {room }}}+1}\right)} C_{j}\left(h_{\text {room }}, t\right) .
\end{aligned}
$$

Substituting (11) in the sigmoid function $\left(c_{j}(z, t)\right)$ definition and computing the space averaged concentration between 0 and $h_{\text {out }}$, we have that:

$$
C_{j}\left(h_{\text {out }}, t\right)=\frac{\beta_{j}^{k} h_{\text {room }}}{\ln \left(\frac{e^{\beta_{j}^{k}\left(1-\gamma_{j}^{k}\right) h_{\text {room }}}+1}{e^{-\beta_{j}^{k} \gamma_{j}^{k} h_{\text {room }}+1}}\right)} C_{j}\left(h_{\text {room }}, t\right) \frac{1}{h_{\text {out }}} \int_{0}^{h_{\text {out }}} \frac{1}{1+e^{-\beta_{j}^{k}\left(\theta-\gamma_{j}^{k} h_{\text {room }}\right)}} d \theta .
$$


Finally, substituting the expression of $C_{j}\left(h_{\text {out }}, t\right)$ in equation (10) we obtain:

$$
\begin{aligned}
\dot{C}_{j}\left(h_{\text {room }}, t\right) & =\frac{\dot{m}_{j, \text { in }}(t)}{V_{R}}+\frac{u_{\text {in }}(t) S_{i n} c_{j, a t m}}{V_{R}}-\frac{u_{\text {in }}(t) S_{\text {in }}}{V_{R}} \frac{\beta_{j}^{k} h_{\text {room }}}{\ln \left(\frac{e^{\beta_{j}^{k}\left(1-\gamma_{j}^{k}\right) h_{\text {room }}}+1}{e^{-\beta_{j}^{k} \gamma_{j}^{k} h_{\text {room }}}+1}\right)} C_{j}\left(h_{\text {room }}, t\right) \times \\
& \times \frac{1}{h_{\text {out }}} \int_{0}^{h_{\text {out }}} \frac{1}{1+e^{-\beta_{j}^{k}\left(\theta-\gamma_{j}^{k} h_{\text {room }}\right)}} d \theta .
\end{aligned}
$$

The simplified model for the secondary system is then the continuous time non-linear delay system:

$$
\begin{aligned}
\dot{C}_{j}\left(h_{\text {room }}, t\right)= & \frac{\dot{m}_{j, i n}(t)}{V_{R}}+\frac{S_{\text {in }} c_{j, a t m} \eta}{V_{R}} u_{\text {fan }}\left(t-\delta_{t}(t)\right) \\
& -\frac{S_{i n} \eta}{V_{R}} u_{\text {fan }}\left(t-\delta_{t}(t)\right) \frac{h_{\text {room }} \ln \left(\frac{e^{\beta_{j}^{k}\left(h_{\text {out }}-\gamma_{j}^{k} h_{\text {room }}\right)}+1}{e^{-\beta_{j}^{k} \gamma_{j}^{k} h_{\text {room }}}+1}\right)}{h_{\text {out }} \ln \left(\frac{e^{\beta_{j}^{k}\left(1-\gamma_{k}\right) h_{\text {room }}}+1}{e^{-\beta_{j}^{k} \gamma_{j}^{k} h_{\text {room }}}+1}\right)} C_{j}\left(h_{\text {room }}, t\right),
\end{aligned}
$$

where $\delta_{t}$ is the worst-case delay introduced by the tarpaulin tube $\tau_{\text {tarp }}(t)$, as defined in equation (1). The dynamics becomes affine if we consider the control input $u_{\text {fan }}$ piecewise constant (i.e. constant during the simulation time step) as we will do in the next subsection. Note that $\eta$ depends on the airflow velocity in the tube, due to the Reynolds number dependency.

\subsection{Control design}

Given the model of the secondary system (13), we define the following control specifications.

1. Safety: the gas concentrations can not enter an unsafe set (a red alert zone), given by standard acceptable air quality for humans.

2. Comfortable air quality: the gas concentrations might enter, only for a bounded amount of time, a set (a yellow alert zone) that satisfies safety for humans, but might be uncomfortable.

These two properties intuitively state the following: we guarantee that the oxygen concentration is always over the minimum safety threshold for humans, and we require that if a disturbance (e.g. the entrance of a truck in a room) moves it under a threshold of optimality (comfortable air quality), then it only happens for a short amount of time.

Our aim is to automatically verify whether the controlled system satisfies the aforementioned safety/comfort properties. Thus, we first need to model the two specifications as formulae. The first strong specification (classical safety property) can be modeled using the temporal logic CTL [37], while the second relaxed specification can be modeled using the temporal logic TCTL [38]. Clearly, we have to verify that both of them are satisfied.

Because of the hybrid nature of the problem (e.g. number of trucks in an extraction room), it is extremely hard to verify exhaustively on the model (13) whether the specifications above are satisfied for any discrete and continuous disturbance. For this reason, we first obtain a conservative hybrid model with affine dynamics of our closed-loop system, then construct a timed automaton abstraction of the hybrid automaton, which preserves CTL and TCTL 
temporal properties. Timed automata can generally be abstracted into finite state systems [39], which renders the model checking decidable.

We now define a hybrid model of our closed-loop system with affine dynamics thanks to the following assumptions.

Assumption 1. As a first approach, we consider only two speed levels (which is the case in some of the actual mines, where only basic automation is available), namely:

$$
\dot{\theta}_{\text {fan }} \in\left\{\dot{\theta}_{\text {fan }}^{L}, \dot{\theta}_{\text {fan }}^{H}\right\} .
$$

Depending on the maximum gas emission/consumption of the trucks, we can chose a desired airflow $u_{i n}^{H}$ at the endpoint of the tarpaulin tube and derive the corresponding fan velocity $\dot{\theta}_{\text {fan }}^{H}$ using appropriate engineering data sheet.

Assumption 2. A threshold control strategy is considered: we can switch fans to low and high speed levels when the gas concentrations hit some given thresholds.

Assumption 3. The WSN design guarantees that the measured concentration is affected by a bounded estimation error $\varepsilon>0$ and that the estimation and communication delays are bounded by a value $\delta_{c}>0$. As discussed in Section 1, the main wiring problem in the mine is in the extraction room. Thus, we reasonably assume that the threshold is checked on a gateway sensor positioned in the room and that the control signal is transmitted to the fan on a cable (see Fig. 11). On the basis of these assumptions, the secondary control system can be

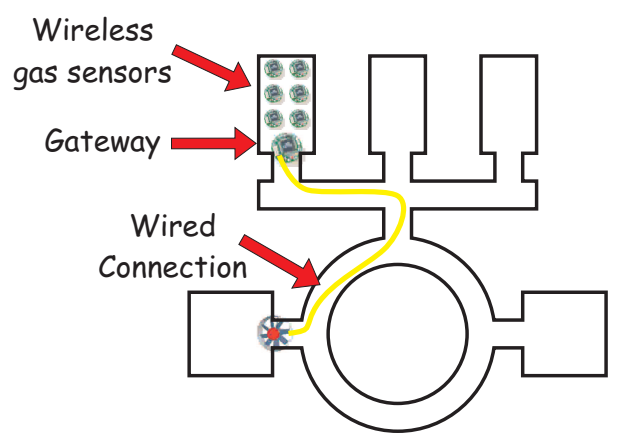

Figure 11. Proposed network architecture for control.

modeled as a non deterministic affine hybrid automaton. Non deterministic guard conditions determined by $\varepsilon>0$ model the estimation error, while a clock variable models estimation, communication and actuation delays $\delta=\delta_{c}+\delta_{t}$.

We consider a three dimensional continuous state space $x(t) \in \mathbb{R}^{3}$, where the first component is the average concentration of oxygen $x_{1}(t)=c_{O_{2}}(t)$, the second is the concentration of carbon monoxide $x_{2}(t)=c_{C O}(t)$, and the third is the concentration of carbon dioxide $x_{3}(t)=c_{C_{2}}(t)$ in the extraction room. The dynamics of this three dimensional system is obtained by replying the differential equation (12) for each gas:

$$
\dot{x}_{i}(t)=a_{i} x_{i}(t)+b_{i}, i \in\{1,2,3\},
$$

with the coefficients $\left\{a_{i}, b_{i}\right\}$ obtained according to (13). The constants $a_{i}$ depend on the buoyancy characteristics, on the input volumetric airflow and on the concentration of the $i^{\text {th }}$ 
gas in the room, while the constants $b_{i}$ depend on the $i^{t h}$ gas emission/consumption of the trucks and on the input volumetric airflow.

Since we have two speed levels, each one of the constants $a_{i}, b_{i}$ can assume two values (respectively $a_{i}^{L}, a_{i}^{H}$ and $b_{i}^{L}, b_{i}^{H}$ ). Let $g_{l, 1}, g_{h, 2}, g_{h, 3}$ be the thresholds on the gas concentrations that trigger the increase of the fan speed, and $g_{h, 1}, g_{l, 2}, g_{l, 3}$ be the thresholds on the gas concentrations that trigger the decrease of the fan speed. In our hybrid model of the controlled secondary system we consider the worst case value for the disturbance introduced by the trucks, namely when there are two working trucks in the extraction room. The hybrid model (depicted in Fig. 12) is now described, using the notations introduced in [35].

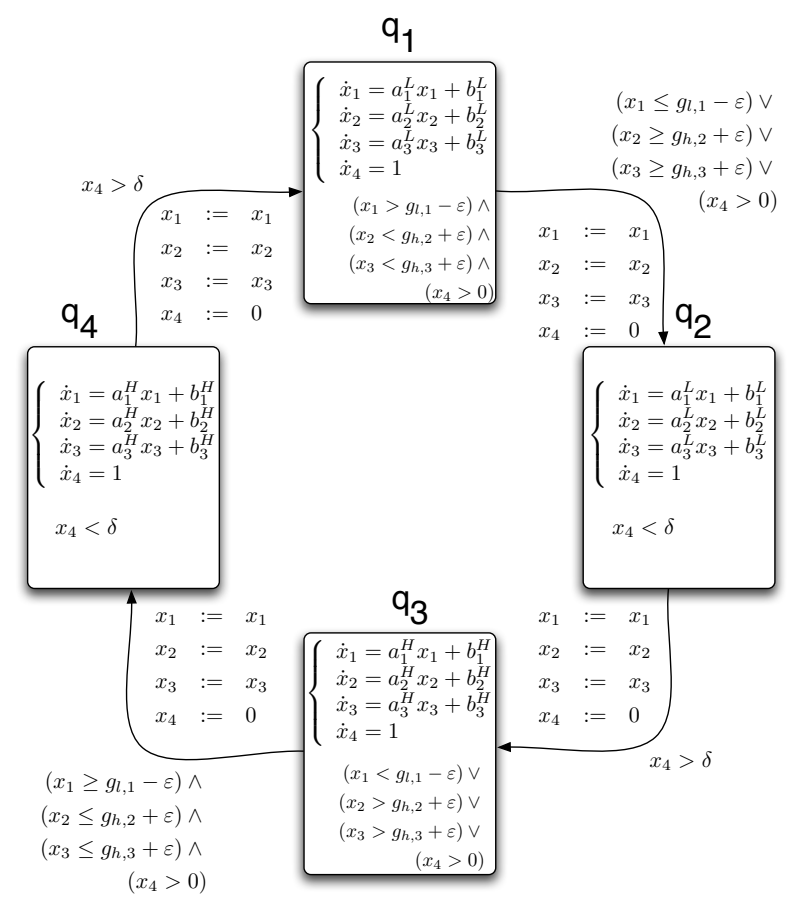

Figure 12. Hybrid model of the closed-loop secondary system.

- $Q=\left\{q_{1}, q_{2}, q_{3}, q_{4}\right\}$ is the set of discrete states that models the two speed levels of the fan and the two actuation delays when we switch from one speed to the other and viceversa. In particular, $q_{1}$ models the low speed state, $q_{2}$ models the low to high switch delay, $q_{3}$ models the high speed state and $q_{4}$ models the high to low switch delay.

- $X=\mathbb{R}^{4}$ where $x_{1}, x_{2}$ and $x_{3}$ represent, respectively, the concentration of oxygen, carbon monoxide and carbon dioxide, while $x_{4}$ is a clock state variable.

- Init $=\left\{\left(q_{1}, x_{0}\right)\right\}$ is the set of initial conditions.

- $E=\left\{\left(q_{1}, q_{2}\right),\left(q_{2}, q_{3}\right),\left(q_{3}, q_{4}\right),\left(q_{4}, q_{1}\right)\right\}$ is the set of discrete transitions. 
- The continuous dynamics for the discrete states $q_{1}, q_{2}$ is given by:

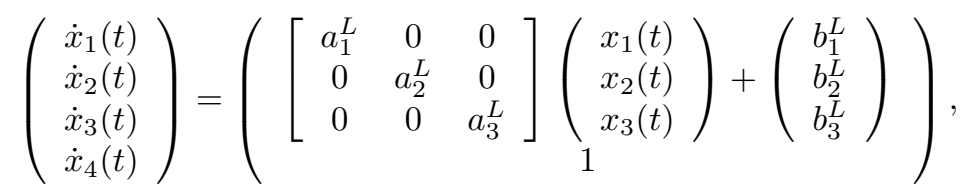

and the continuous dynamics for the discrete states $q_{3}, q_{4}$ is given by:

$$
\left(\begin{array}{c}
\dot{x}_{1}(t) \\
\dot{x}_{2}(t) \\
\dot{x}_{3}(t) \\
\dot{x}_{4}(t)
\end{array}\right)=\left(\left[\begin{array}{ccc}
a_{1}^{H} & 0 & 0 \\
0 & a_{2}^{H} & 0 \\
0 & 0 & a_{3}^{H}
\end{array}\right]\left(\begin{array}{c}
x_{1}(t) \\
x_{2}(t) \\
x_{3}(t)
\end{array}\right)+\left(\begin{array}{c}
b_{1}^{H} \\
b_{2}^{H} \\
b_{3}^{H}
\end{array}\right)\right)
$$

- Invariant, guard and reset are defined as follows:

$$
\begin{aligned}
& \text { Inv }\left(q_{1}\right)=\left\{x \in \mathbb{R}^{4} \mid x_{1}>g_{l, 1}-\varepsilon \wedge x_{2}<g_{h, 2}+\varepsilon \wedge x_{3}<g_{h, 3}+\varepsilon \wedge x_{4}>0\right\}, \\
& \text { Inv }\left(q_{2}\right)=\left\{x \in \mathbb{R}^{4} \mid x_{4}<\delta\right\}, \\
& \text { Inv }\left(q_{3}\right)=\left\{x \in \mathbb{R}^{4} \mid x_{1}<g_{h, 1}+\varepsilon \vee x_{2}>g_{l, 2}-\varepsilon \vee x_{3}>g_{l, 3}-\varepsilon \vee x_{4}>0\right\}, \\
& \text { Inv }\left(q_{4}\right)=\left\{x \in \mathbb{R}^{4} \mid x_{4}<\delta\right\} \\
& G\left(q_{1}, q_{2}\right)=\left\{x \in \mathbb{R}^{4} \mid x_{1} \leqslant g_{l, 1}-\varepsilon \vee x_{2} \geqslant g_{h, 2}+\varepsilon \vee x_{3} \geqslant g_{h, 3}+\varepsilon\right\}, \\
& G\left(q_{2}, q_{3}\right)=\left\{x \in \mathbb{R}^{4} \mid x_{4} \geqslant \delta\right\}, \\
& G\left(q_{3}, q_{4}\right)=\left\{x \in \mathbb{R}^{4} \mid x_{1} \geqslant g_{h, 1}+\varepsilon \wedge x_{2} \leqslant g_{l, 2}-\varepsilon \wedge x_{3} \leqslant g_{l, 3}-\varepsilon\right\}, \\
& G\left(q_{4}, q_{1}\right)=\left\{x \in \mathbb{R}^{4} \mid x_{4} \geqslant \delta\right\} \\
& \forall e \in E, R(e, x(t))=\left(x_{1}(t), x_{2}(t), x_{3}(t), 0\right)^{\prime} .
\end{aligned}
$$

The threshold control strategy is designed by defining the guards $g_{l, 1}, g_{l, 2}, g_{l, 3}, g_{h, 1}, g_{h, 2}$ and $g_{h, 3}$. We now automatically verify, by using the theoretical results introduced in [36] and the tool developed in [35], if for a given control strategy the hybrid automaton defined above (which models the closed-loop secondary system according to our control strategy) satisfies Safety and Comfort properties. More precisely, define the Safety specification introduced above as a CTL formula $\psi_{s}$, and define the comfortable air quality specification as a TCTL formula $\psi_{c}\left(t_{\max }\right)$, where $t_{\max }$ is the maximum time the system is allowed to dwell in uncomfortable air quality.

Unfortunately, model checking is in general undecidable also for affine hybrid automata: an important technique used to cope with complexity is abstraction. In recent papers [35, 36] an algorithm that constructs an abstraction of a hybrid automaton with affine dynamics was proposed and implemented. Such algorithm preserves temporal properties expressed by CTL and TCTL temporal logics. The abstract model belongs to a subclass of timed automata, which is called durational graph. Durational graphs are a special class of timed and hybrid automata $[40,41]$, in which the continuous variables are clocks, increasing with a constant slope. Resets are restricted to clock resets to 0 .

The following result is directly implied by the results developed in [36]:

Proposition 1. Given a hybrid automaton $\mathcal{H}$ and a durational graph $\mathcal{G}$ such that $\mathcal{H}$ and $\mathcal{G}$ are bisimilar with precision $\varphi\left(\mathcal{H} \approx_{\varphi} \mathcal{G}\right)$, then:

i. If the specification $\psi_{s}$ is satisfied for the system $\mathcal{G}$, then it is satisfied for the system $\mathcal{H}$;

ii. If the specification $\psi_{c}\left(t_{\max }-\varphi\right)$ is satisfied for the system $\mathcal{G}$, then $\psi_{c}\left(t_{\max }\right)$ is satisfied for the system $\mathcal{H}$. 
According to the above proposition, we can verify properties of the affine hybrid automaton by checking properties on the durational graph abstraction, using model checking tools for timed automata (e.g. KRONOS [42], UPPAAL [43]). If for an initial choice of the threshold the system is not verified to be safe, we can iteratively run the verification procedure for tighter thresholds until safety and comfort are both satisfied.

The abstraction algorithm's graphical output is illustrated in Fig. 13(a). The algorithm intuitively works as follows. The set of initial conditions of the gas concentrations (blue polytope) is partitioned in a finite number of polytopes according to the continuous dynamics and the guard sets. The property of each element of the partition is that, for each pair of initial conditions $x_{1}^{0}, x_{2}^{0}$ belonging to it, the arrival times $t_{1}, t_{2}$ to the guard satisfy $\left|t_{1}-t_{2}\right|<\varphi$. This procedure is iterated to all guard sets (green and red polytopes) of the hybrid automaton. Each partition element is translated into a discrete state of the abstracting durational graph. The resulting durational graph $\mathcal{G}$ satisfies $\mathcal{H} \approx_{\varphi} \mathcal{G}$, and thus Proposition 1 holds. Moreover, we can get from the precision $\varphi$ of our abstraction a measure of safety: this can be used to optimize the thresholds by iterative search, in order to guarantee safety with a desired robustness and energy consumption reduction.

By executing the automatic verification procedure on our hybrid model using the thresholds $g_{l, 1}=0.2955, g_{l, 2}=0.5 \cdot 10^{-3}, g_{l, 3}=0.0885, g_{h, 1}=0.2975, g_{h, 2}=2.5 \cdot 10^{-3}, g_{h, 3}=0.091$ (in $\left.\left[\mathrm{Kg} / \mathrm{m}^{3}\right]\right)$ and an abstraction precision $\varphi=1 \mathrm{~s}$, we verified on the abstraction $\mathcal{G}$ that $\psi_{s}$ is satisfied, and $\psi_{c}\left(t_{\max }\right)$ is satisfied for $t_{\max }=61 \mathrm{~s}$. Since our abstraction has been constructed with precision $\varphi=1 s$, then Proposition 1 implies that (1) the original is safe and (2) the maximum time of uncomfortable air quality is bounded by $62 \mathrm{~s}$.

For completeness, we also show in Fig. 13(b) some Matlab simulations of the closed loop system. For the particular initial condition chosen for this simulation, safety and air quality can be verified using the plots. The advantage of automatic verification is that the same two properties can be verified in one shot for the whole set of initial conditions, which has infinite cardinality.

\section{COMPARISON BETWEEN THE TWO APPROACHES}

\subsection{Comparison framework}

In this section we compare the continuous and the hybrid control strategies on the test case proposed in Section 3.5. The safety thresholds $\overline{C O}=0.000234 \mathrm{~kg} \cdot \mathrm{m}^{3}$ and $\overline{N O}=0.0059 \mathrm{~kg} \cdot \mathrm{m}^{3}$ are considered as strong constraints, in the sense that the regulated pollutant concentrations have to remain below these values at any time. The comparison is based on simulation results, for which we compare regulation efficiency, energy consumption, robustness with respect to model uncertainties, and computational complexity.

For comparison consistency, the MPC control law depicted in 4.1 is tested with a control regulation objective set to $90 \%$ of the thresholds, while for the discrete strategy a choice of the guards $g_{l, C O / N O}$ and $g_{h, C O / N O}$ at $80 \%$ and $90 \%$ of the safety thresholds is made. For both strategies, the high and low operating conditions of the fans result in flow speeds $u_{f a n}$ of $10 \mathrm{~m} / \mathrm{s}$ and $80 \mathrm{~m} / \mathrm{s}$, respectively. The parameters for the MPC strategy are set to $\lambda=10^{-5}$, $N=100$ and $N_{u}=1$. Simulation results of the two strategies are depicted in Fig. 14 . 


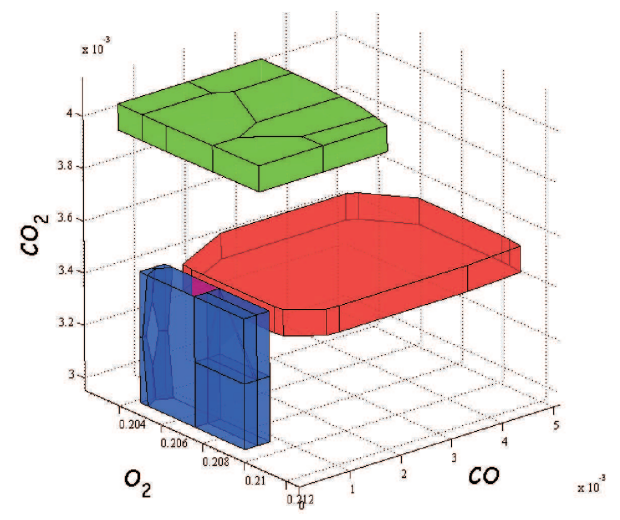

(a) Polytopes representing the set of initial conditions and the projections of their executions with the guard sets
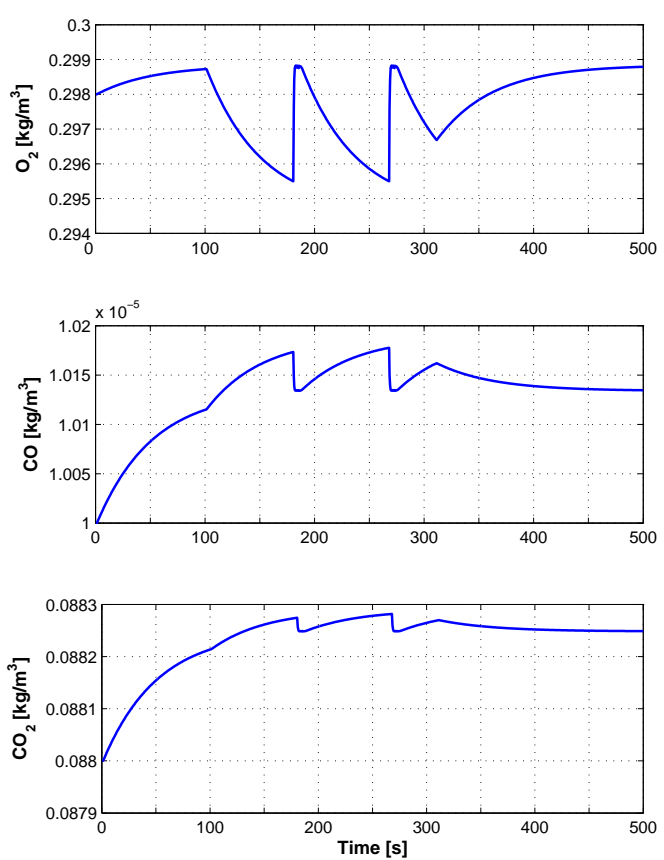

(b) Temporal simulation

Figure 13. Matlab ${ }^{T M}$ simulations and screenshot of the abstraction algorithm output.

\subsection{Regulation Efficiency}

For both strategies, the safety conditions are satisfied. The MPC is tuned such that both approaches have a comparable energy consumption. We can see that, in terms of regulation efficiency, the MPC leads most of the time as the pollutant concentration is significantly lower (40\% lower for the one truck case, equivalent when there are 2 trucks and $12 \%$ higher for the three trucks case).

\subsection{Energy Consumption and control input}

The energy consumption is compared using the fan model discussed in Section 3.4. Fig. 14 shows that the MPC strategy is more efficient (by approximately $8.2 \%$ ) with respect to energy consumption. This is mainly due to the fact that a relatively high $\lambda$ has been chosen for this comparison, leading to a strong weight on power consumption optimization. Furthermore, the MPC approach obviously leads to smoother control laws. Note that the observed differences between the two approaches are also related to the nonlinear properties of the plant and to 

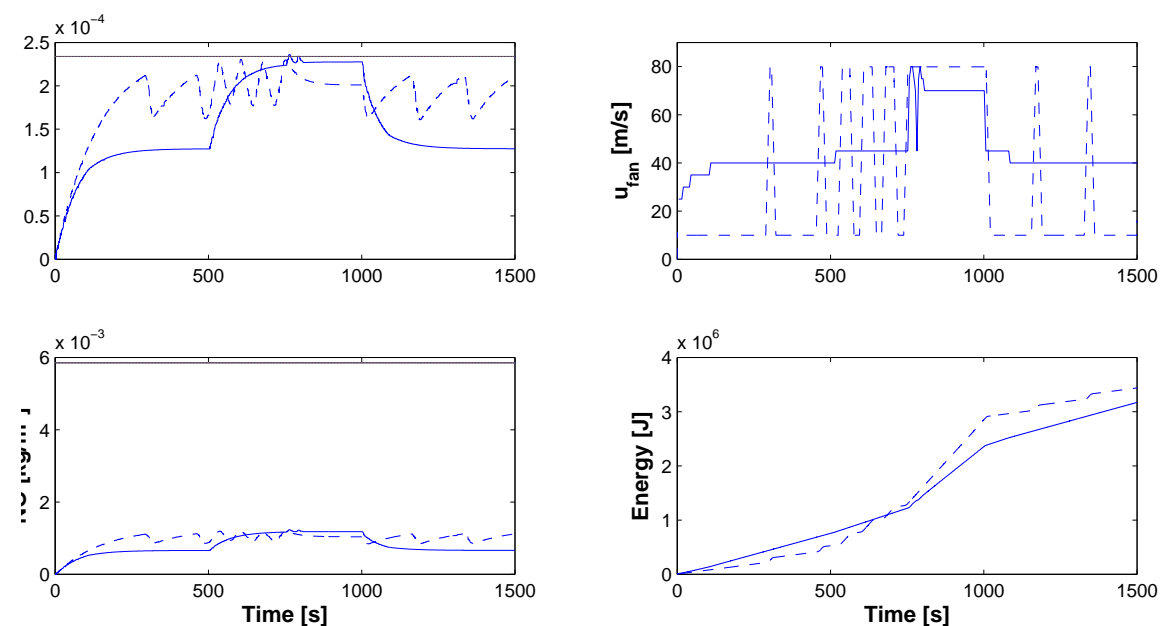

Figure 14. Comparison of the two approaches - MPC (solid) and Hybrid (dotted)

the tuning of the controller gains (or choice of the guards for the thresholds approach).

\subsection{Robustness with respect to model uncertainties}

In the receding horizon strategy, one has to consider a prediction model of the system. In our case, the prediction model is the reference model, with the maximum time delay, and prediction of pollutant sources at mid value. Feasibility of successive optimization problems appears to be a crucial point in the receding horizon strategy. The constraints are taken into account by adding some hard penalization terms in the optimization criterion. Thus, when the constraints begin to be slightly violated, the control law increases its value to satisfy the constraints as soon as possible. More advanced strategies are investigated in [44], but the previous approach appears to be sufficient for the mine ventilation, as illustrated by the robustness tests presented in Fig. 15.

Two different robustness tests were performed. In the first one (Fig. 15(a)), we consider underestimated values for the prediction of the pollutant sources. Thus, for this test, the prediction model assumes that there is only one truck in the mine. This is a worst case situation, as can be seen from the real truck profile of Fig. 7. The second test (Fig. 15(b)) consists of testing a $20 \%$ underestimated gain of the system. In other words, the prediction model assumes that the amount of pollutant is always lower and slower than in reality. Both tests show that the MPC strategy is robust against pollutant prediction errors and model uncertainties.

While the hybrid approach is structurally robust, in the sense that it converges in finite time, the thresholds may not be satisfied due to the system inertia and neglected dynamics. This can happen, for example, if we choose the guards at $85 \%$ and $95 \%$ of the threshold values, as illustrated in Fig. 16. Even if the fans are set to their maximum value once the threshold is triggered, the controller can not predict the safety margin that is necessary to compensate 

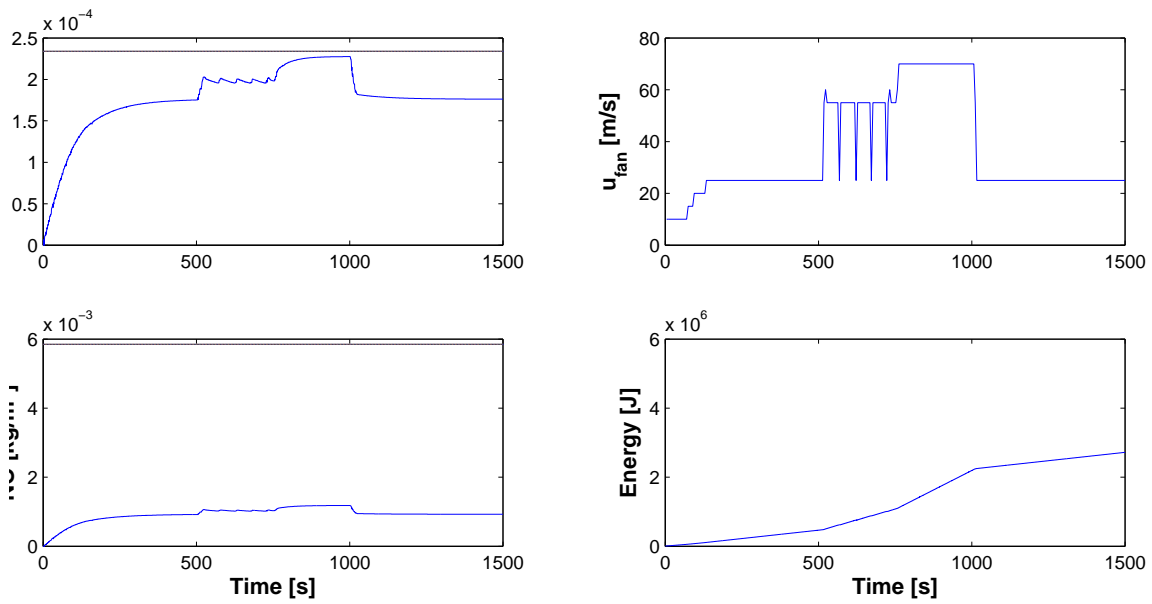

(a) MPC with underestimated pollutant source (1 truck)
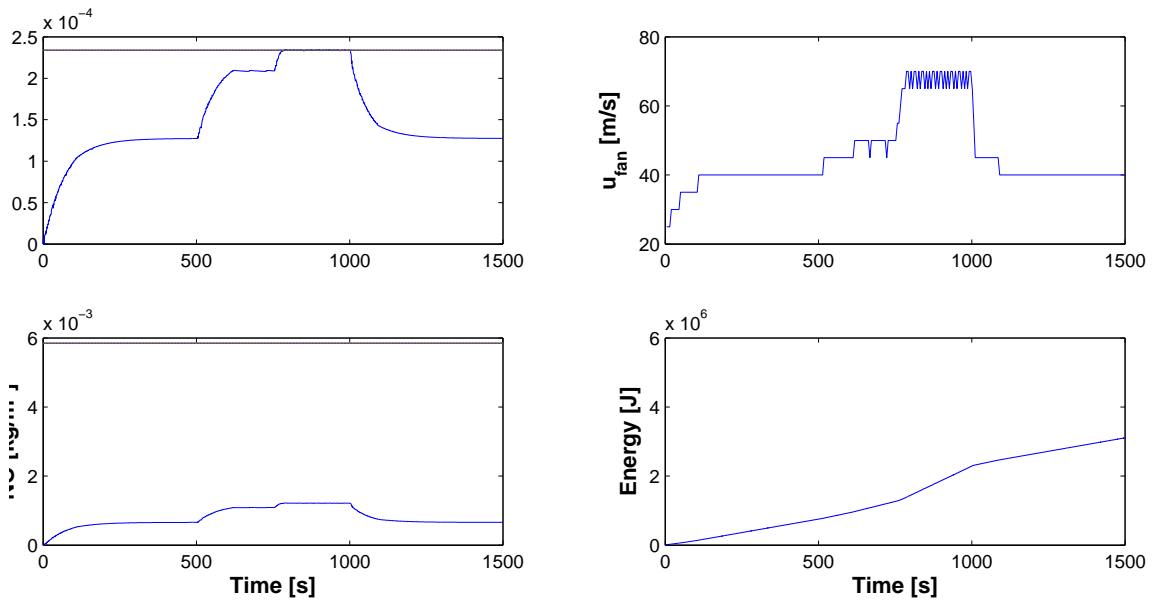

(b) MPC with parameter uncertainty

Figure 15. Robustness tests $\left(N=100 s, N_{u}=1, \lambda=10^{-5}\right)$.

for the inertia of the pollutants concentration in the room.

\subsection{Computational Complexity}

The computational complexity of the threshold control strategy is $O(1)$, namely the control algorithm can be executed in a constant (and very short) time. In fact, the controller is very simple and does not require any kind of computation, since it is based only on the comparison between measurements and safety thresholds. On the contrary, the MPC approach 

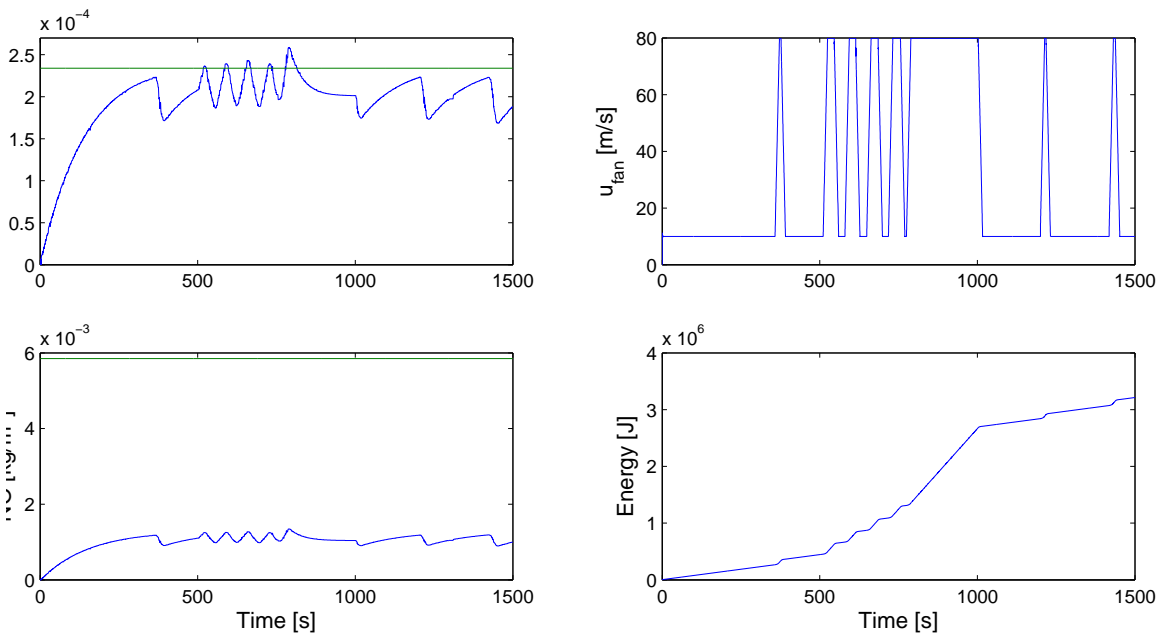

Figure 16. Threshold control strategy with slightly modified guards

is computationally time consuming, since hard optimization problems have to be solved at each sampling time. More precisely, the evaluation of the cost function implies the simulation of the whole model, as well as the numerical integration of differential equations. The number of such simulations grows exponentially with the number of degrees of freedom $N_{u}$ in the control law design. Further, in the real implementation of the MPC control algorithm it is necessary to take into account, before the prediction stage, the reconstruction of those states which parameterize the finite time optimal control problem to be solved at each sampling period.

Remark 3. The comparison between the continuous and the hybrid control strategies have shown that the nonlinear model predictive control outperforms the safety control, in terms of energy consumption minimization and robustness. This performance is achieved at the price of a higher computational complexity, which renders the MPC difficult to envision for embedded systems, while the threshold control strategy can be included in a modern wireless sensor equipped with a microchip. From an implementation point of view we can then relate the choice of the control strategy to the regulation architecture presented in Section 2.1. Indeed, if a centralized approach can be implemented, then there is no difficulty to set the MPC in the control room. If the computational resource is limited to the WSN, then the threshold control strategy can be used to reduce the energy consumption in comparison with the actual on/off strategy.

\section{CONCLUSIONS}

We provided a precise description of the mining ventilation problem, a novel industrial case in the field of control that is of prime environmental importance and where advanced control methodologies can bring a major improvement. This problem was formulated as the 
one of wireless ventilation control for large-scale systems, the core idea being to combine WSN capabilities with model-based control strategies. We consequently derived a new control-oriented model that includes the various dynamics of this multi-physics process. The constraints induced by the WSN were mostly associated with time-delays and packet losses. The modeling of the complete system have shown that the communication constraints have a secondary importance compared to the regulated pollutants dynamics.

Two different control approaches were applied: a continuous approach based on a receding horizon scheme (MPC) and a threshold control strategy that uses a mathematical abstraction of the system. The first approach was shown to allow for a precise control of the pollution level, explicitly taking into account the non linearities and operation constraints, and providing for an easy tuning between closed-loop performance and energy consumption. The second approach was focused on guaranteeing safety and providing for a reduced-complexity regulation that is suitable for embedded control. Comparing the two approaches, it appeared that significantly improved performances can be achieved thanks to MPC while the threshold control strategy can be easily embedded in WSN. Such result can be used as an automation design argument, to motivate a centralized control approach (offering larger computational capabilities) or to evaluate the gains associated with a WSN-embedded control solution.

The on-line estimation of key model parameters and the design of a new control strategy that can synthesize the advantages of the two approaches may provide interesting guidelines for future research.

\section{Acknowledgements}

The authors greatly acknowledge the contribution of the anonymous reviewers, who significantly participated in the paper improvement thanks to their thoughtful and constructive remarks. 


\section{REFERENCES}

1. C. van der Merwe, "Mining industry challenged to cut energy consumption $15 \%$ by 2015," Mining weekly online, 1 Jun 2007.

2. X. Inc., "United states industrial electric motor systems market opportunities assessment," Office of energy efficiency and renewable energy, U.S. Department of Energy, Tech. Rep., 1998.

3. P. E. Scheihing, M. Rosenberg, M. Olszewski, C. Cockrill, and J. Oliver, "United states industrial motordriven systems market assessment: Charting a roadmap to energy savings for industry," in International Workshop on Industrial Energy Efficiency Policies: Understanding Success and Failure, Utrecht, The Netherlands, Jun. 1998

4. H. L. Hartmanand, J. M. Mutmansky, R. V. Ramani, and Y. J. Wang, Mine Ventilation and Air Conditioning, $3^{\text {rd }}$ ed. Wiley-Interscience, Oct. 1997.

5. "The second international conference on wireless communications in underground and confined areas," Val-dOr, Canada, Aug. 2008.

6. A. Isaksson, M. Strand, M. D. Di Benedetto, A. D’Innocenzo, F. Santucci, E. Serra, S. Tennina, U. Tiberi, A. Bemporad, S. Di Cairano, F. Maggi, M. Synekidou, M. D'Angelo, K. H. Johansson, A. Seuret, E. Witrant, and C. Fischione, "Final report on the industrial test case," in HYCON Deliverable D4d.5.3, October 2008

7. L. Pomante, F. Santucci, C. Rinaldi, S. Tennina, and C. Fischione, "Mining ventilation automation: Wireless sensing, communication architecture and advanced services," in IEEE CASE, 2008.

8. E. Witrant, A. D'Innocenzo, A. Isaksson, M. Di Benedetto, K. Johansson, F. Santucci, and M. Strand, "Mining ventilation control: a new industrial case for wireless automation," in Proc. of the IEEE Conference on Automation Science and Engineering (CASE 2008), Washington DC, USA, Aug. 2008.

9. G. Sandou, E. Witrant, S. Olaru, and S. Niculescu, "Receding horizon climate control in metal mine extraction rooms," in IEEE CASE, 2008.

10. M. Di Benedetto, A. D'Innocenzo, E. Serra, and E. Witrant, "Automatic verification of wireless control in a mining ventilation system," in IEEE CASE'08, 2008.

11. S. Tolmachev and E. Fainshtein, "Analysis of multi-branch junctions in ventilation networks, regarded as systems with distributed parameters," Journal of Mining Science, vol. 4(2), pp. 128-133, 1968.

12. F. Klebanov and G. Martynyuk, "A method for experimental determination of the coefficient of longitudinal turbulent diffusion in ventilating currents of mine workings," Journal of Mining Science, vol. 9(4), pp. 413-416, 1973.

13. G. Kalabin, A. Baklanov, and P. Amosov, "Calculating the aerogas dynamics of chamber-like mine workings on the basis of mathematical modeling," Journal of Mining Science, vol. 26(1), pp. 61-73, 1990.

14. N. Petrov, M. Shishkin, V. Dnitriev, and V. Shadrin, "Modeling mine aerology problems," Journal of Mining Science, vol. 28(2), pp. 185-191, 1992.

15. N. Petrov, "Methods of solving problems and designing equipment for mine ventilation," Journal of Mining Science, vol. 30(2), pp. 195-204, 1994.

16. E. Widzyk-Capehart and B. Watson, "Agnew gold mine expansion mine ventilation evaluation using VentSim," in Proc. of the $7^{\text {th }}$ International Mine Ventilation Congress, 2001.

17. D. Estrin, R. Govindan, J. Eidemann, and S. Kumar, "Next century challenges: Scalable coordination in sensor networks," in Proceedings of the ACM/IEEE International Conference on Mobile Computing and Networking, 1999

18. A. Ribeiro and G. B. Giannakis, "Bandwidth-constrained distributed estimation for wireless sensor networkspart I and II," IEEE Transaction on Signal Processing, vol. 54, July 2006.

19. E. Witrant, K. Johansson, and the HynX team, "Air flow modelling in deep wells: application to mining ventilation," in IEEE CASE, 2008

20. W. Schmidt, "Turbulent propagation of a stream of heated air," Z. angew. Math. Mech., vol. 21, pp. 265-278, 351-363, 1941.

21. B. Morton, "Forced plumes," Journal of Fluid Mechanics, vol. 5, no. 1, pp. 151-163, Jan. 1959.

22. Q. Liu and P. Linden, "The fluid dynamics of an underfloor air distribution system," Journal of Fluid Mechanics, vol. 554, pp. 323-341, Apr. 2006.

23. C. Hirsch, Numerical Computation of Internal \& External Flows: the Fundamentals of Computational Fluid Dynamics, $2^{\text {nd }}$ ed. Butterworth-Heinemann (Elsevier), 2007.

24. P. G. Park, "Randomized protocol design in wireless automation," M. Eng. thesis, Royal Institute of Technology (KTH), Stockholm, Sweden, Jan. 2007.

25. E. Witrant, P. G. Park, M. Johansson, K. Johansson, and C. Fischione, "Control over wireless multi-hop networks," in Proc. of the IEEE Conf. on Control Applic., 2007.

26. P. Lee, S. Garimella, and D. Liu, "Investigation of heat transfer in rectangular microchannels," International Journal of Heat and Mass Transfer, vol. 48, p. 16881704, 2005. 
27. E. Witrant, E. Joffrin, S. Brémond, G. Giruzzi, D. Mazon, O. Barana, and P. Moreau, "A control-oriented model of the current profile in tokamak plasma," Plasma Phys. Control. Fusion, vol. 49, pp. 1075-1105, 2007.

28. L. Ljung, System Identification: Theory for the User (2nd Edition). Prentice Hall PTR, 1998.

29. C. Nerguizian, C. Despins, and S. Affs, "Geolocation in mines with an impulse response fingerprinting technique and neural networks," IEEE Transactions on Wireless Communications, vol. 5, no. 3, 2006.

30. A. Chehri, P. Fortier, and P.-M. Tardif, "Frequency domain analysis of uwb channel propagation in underground mines," in 64th IEEE Conference on Vehicular Technology, 2006.

31. P. Krause, Analysis of Electrical Machinery. McGraw-Hill Book Company, 1986.

32. H. Frey and K. Kim, "Comparison of real-world fuel use and emissions for dump trucks fueled with B20 Biodiesel versus petroleum diesel," in 85 th Annual Meeting of the Transportation Research Board -check-, Jan. 2006.

33. J. Maciejowski, Predictive control with constraints. Englewood Cliffs, NJ: Prentice Hall., 2002

34. D. Q. Mayne, J. B. Rawlings, C. Rao, and P. Scokaert, "Constrained model predictive control: stability and optimality," Automatica, vol. 30, pp. 789-814, 2000.

35. A. D'Innocenzo, A. A. Julius, G. J. Pappas, M. D. Di Benedetto, and S. Di Gennaro, "Verification of temporal properties on hybrid automata by simulation relations," in Proceedings of the $46^{\text {th }}$ IEEE Conference on Decision and Control. New Orleans, Louisiana, USA., 12-14 December 2007.

36. A. D'Innocenzo, A. Julius, M. Di Benedetto, and G. Pappas, "Approximate timed abstractions of hybrid automata," in Proceedings of the $46^{\text {th }}$ IEEE Conference on Decision and Control. New Orleans, Louisiana, USA., 12-14 December 2007.

37. E. Clarke, O. Grumberg, and D. Peled, Model Checking. The MIT Press, Cambridge, Massachusetts, 2002 .

38. R. Alur, C. Courcoubetis, and D. Dill, "Model-checking in dense real-time," Information and Computation, vol. 104(1), pp. 2-34, 1993.

39. R. Alur, T. Henzinger, G. Lafferriere, and G. Pappas, "Discrete abstractions of hybrid systems," Proccedings of the IEEE, vol. 88(2), pp. 971-984, July 2000.

40. R. Alur and D. Dill, "A theory of timed automata," Theoretical Computer Science, vol. 126, pp. 183-235, 1994.

41. R. Alur, C. Courcoubetis, N. Halbwachs, T. Henzinger, P. Ho, X. Nicollin, A. Olivero, J. Sifakis, and S. Yovine, "The algorithmic analysis of hybrid systems," Theoretical Computer Science, vol. 138, pp. 3-34, 1995

42. S. Yovine, "Kronos: A verification tool for real-time systems," International Journal of Software Tools for Technology Transfer, Springer-Verlag, vol. 1(1), pp. 123-133, October 1997.

43. K. G. Larsen, P. Pettersson, and W. Yi, "UPPAAL in a nutshell," International Journal on Software Tools for Technology Transfer, vol. 1(1), pp. 134-152, December 1997.

44. G. Sandou and S. Olaru, Hybrid Systems Computation and Control, ser. Lecture Notes in Computer Science. Springer-Verlag Berlin Heidelberg, 2007, vol. 4416, ch. Ant colony and genetic algorithm for constrained predictive control of power systems, pp. 501-514. 